\title{
ON DEFORMATIONS OF PAIRS (MANIFOLD, COHERENT SHEAF)
}

\author{
DONATELLA IACONO AND MARCO MANETTI
}

\begin{abstract}
We analyse infinitesimal deformations of pairs $(X, \mathcal{F})$ with $\mathcal{F}$ a coherent sheaf on a smooth projective variety $X$ over an algebraically closed field of characteristic 0 . We describe a differential graded Lie algebra controlling the deformation problem, and we prove an analog of a Mukai-Artamkin Theorem about the trace map.
\end{abstract}

\section{INTRODUCTION}

Let $\mathcal{F}$ be a coherent sheaf on a smooth projective variety $X$ over an algebraically closed field of characteristic 0; in particular $\mathcal{F}$ admits a finite locally free resolution. Then, it is defined the sheaf trace morphism

$$
\operatorname{Tr}: \mathcal{H} m_{\mathcal{O}_{X}}(\mathcal{F}, \mathcal{F}) \rightarrow \mathcal{O}_{X},
$$

and the trace maps

$$
\operatorname{Tr}^{i}: \operatorname{Ext}_{X}^{i}(\mathcal{F}, \mathcal{F}) \rightarrow H^{i}\left(X, \mathcal{O}_{X}\right),
$$

which are the morphisms induced in hypercohomology by $\operatorname{Tr}$ see e.g. [1, 16, 27].

The maps $\operatorname{Tr}^{1}$ and $\operatorname{Tr}^{2}$ have a clear interpretation in the setting of deformation theory of $\mathcal{F}$ and of the determinant bundle $\operatorname{det} \mathcal{F}$ of $\mathcal{F}$. Every deformation of $\mathcal{F}$ induces naturally

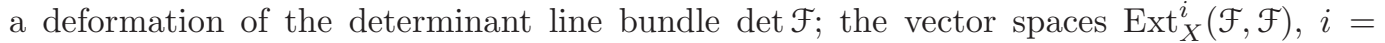
1,2 , are the tangent and obstruction spaces of the functor Def $\mathcal{F}_{\mathcal{F}}$ of deformations of $\mathcal{F}$; the spaces $H^{i}\left(X, \mathcal{O}_{X}\right), i=1,2$, are the tangent and obstruction spaces of the functor $\operatorname{Def}_{\operatorname{det}} \mathcal{F}$ of deformations of $\operatorname{det} \mathcal{F}$. Then, the maps $\operatorname{Tr}^{i}, i=1,2$, are induced by the natural transformation $\operatorname{Def}_{\mathcal{F}} \rightarrow \operatorname{Def}_{\operatorname{det} \mathcal{F}}$.

Moreover, since we are in characteristic 0 , the Picard functor is unobstructed and this allows to prove that $\operatorname{Tr}^{2}$ annihilates all the obstructions to deformations of $\mathcal{F}$. As a consequence, we have the following well known result.

Theorem 1.1 (Mukai-Artamkin $[1,22]$ ). In the above situation, if $\operatorname{Tr}^{2}$ is injective, then the deformation functor $\operatorname{Def}_{\mathcal{F}}$ is unobstructed. If $\operatorname{Tr}^{1}$ is surjective and $\operatorname{Tr}^{2}$ is injective, then the natural transformation $\operatorname{Def}_{\mathcal{F}} \rightarrow \operatorname{Def}_{\operatorname{det} \mathcal{F}}$ is smooth.

In their papers, Mukai and Artamkin assume that $\mathcal{F}$ is simple, however it is immediate to see that this assumption is used only for the pro-representability of the functor $\operatorname{Def}_{\mathcal{F}}$, and hence for the existence of the universal deformation of $\mathcal{F}$, while the proof of the smoothness of the semiuniversal deformation works also without the simpleness assumption.

One of the main goal of this paper is to extend the previous result to the case of infinitesimal deformations of pairs $(X, \mathcal{F})$, consisting of deformations of both the variety $X$ and the coherent sheaf $\mathcal{F}$.

As a first result, we describe a well defined homotopy class of differential graded Lie algebras associated with the deformations of the pair $(X, \mathcal{F})$.

Date: June 20, 2018.

2010 Mathematics Subject Classification. 14D15, 17B70, 18G50, 13D10.

Key words and phrases. Deformation of manifold and coherent sheaf, differential graded Lie algebras. 
For every quasi-coherent sheaf of DG-Lie algebras $\mathcal{L}^{*}$ on an algebraic variety $X$ it is defined, up to homotopy equivalence, the DG-Lie algebras of derived sections $R \Gamma\left(X, \mathcal{L}^{*}\right)$ and we shall say that a deformation problem is controlled by $\mathcal{L}^{*}$ if it is controlled in the usual way by the DG-Lie algebra $R \Gamma\left(X, \mathcal{L}^{*}\right)$. A canonical representative for $R \Gamma\left(X, \mathcal{L}^{*}\right)$ is given by the totalization, in the simplicially enriched model category of DG-Lie algebras, of the cosimplicial space of Cech cochains of $\mathcal{L}^{*}$, with respect to any open affine cover of $X$, see Section 6 .

For a coherent sheaf $\mathcal{F}$ on a projective smooth variety $X$, it is known that the deformations of $\mathcal{F}$ are controlled by the sheaf of DG-Lie algebra of endomorphisms of any finite locally free resolution of $\mathcal{F}[6]$.

It is also known that if $\mathcal{E}$ is a locally free sheaf on a smooth variety $X$, then the deformations of the pair $(X, \mathcal{E})$ are controlled by the sheaf of Lie algebra of first order differential operators on $\mathcal{E}$ with principal symbol [13, 20,25]. For a general coherent sheaf $\mathcal{F}$, the sheaf of differential operators on $\mathcal{F}$ is equally well defined [9, Section 16.8] but, in general, it is not the right object controlling the deformations of pairs.

Therefore, we first introduce the right algebraic object associated with the deformation problem: we shall call this object the module of derivations of pairs. It extends the sheaf of differential operators on $\mathcal{F}$, involving a finite locally free resolution of $\mathcal{F}$. This allows us to define a coherent sheaf of DG-Lie algebras over $X$ controlling the deformations of the pair $(X, \mathcal{F})$ (Theorem 7.11).

In particular, the cohomology groups $T_{(X, F)}^{i}$ of the associated DG-Lie algebra fits into a long exact sequence:

$$
\cdots \rightarrow \operatorname{Ext}_{X}^{i}(\mathcal{F}, \mathcal{F}) \rightarrow T_{(X, \mathcal{F})}^{i} \rightarrow H^{i}\left(X, \Theta_{X}\right) \rightarrow \operatorname{Ext}_{X}^{i+1}(\mathcal{F}, \mathcal{F}) \rightarrow \cdots,
$$

where $\Theta_{X}$ denotes the tangent sheaf of $X$. In particular, we recover the well known fact that if $\operatorname{Ext}_{X}^{2}(\mathcal{F}, \mathcal{F})=0$, then the natural transformation $\operatorname{Def}_{(X, \mathcal{F})} \rightarrow \operatorname{Def}_{X}$ is smooth, since it is surjective on tangent spaces $\left(T_{(X, \mathcal{F})}^{1} \rightarrow H^{1}\left(X, \Theta_{X}\right)\right)$ and injective on obstruction spaces $\left(T_{(X, \mathcal{F})}^{2} \rightarrow H^{2}\left(X, \Theta_{X}\right)\right)$.

Then, we devote our attention to the natural transformations

$$
\operatorname{Def}_{(X, \mathcal{F})} \rightarrow \operatorname{Def}_{(X, \operatorname{det} \mathcal{F})} \rightarrow \operatorname{Def}_{X} .
$$

In particular, we describe an extension of the trace maps to the DG-Lie algebra of differential operators with principal symbol (Theorem 5.6).

Finally, we are able to prove the following result (Theorem 7.16), which is the analog of Theorem 1.1, for deformations of pairs.

Theorem 1.2. Let $\mathcal{F}$ be a coherent sheaf on a projective smooth variety $X$ defined over an algebraically closed field of characteristic 0. Consider the trace maps $\operatorname{Tr}^{i}: \operatorname{Ext}_{X}^{i}(\mathcal{F}, \mathcal{F}) \rightarrow$ $H^{i}\left(X, \mathcal{O}_{X}\right)$ :

(1) if the map $\operatorname{Tr}^{1}$ is surjective and the map $\operatorname{Tr}^{2}$ is injective, then the natural transformation $\operatorname{Def}_{(X, \mathcal{F})} \rightarrow \operatorname{Def}_{(X, \operatorname{det} \mathcal{F})}$ is smooth;

(2) if $\operatorname{Tr}^{2}$ is injective and $\operatorname{Def}_{(X, \operatorname{det} \mathcal{F})}$ is unobstructed, then $\operatorname{Def}_{(X, \mathcal{F})}$ is unobstructed;

(3) if $H^{0}\left(X, \Theta_{X}\right)=0$ and $\operatorname{Ext}_{X}^{0}(\mathcal{F}, \mathcal{F})=\mathbb{K}$ (e.g., if $\mathcal{F}$ is simple), then $\operatorname{Def}_{(X, \mathcal{F})}$ is pro-representable.

For instance if $\mathcal{F}$ is a simple coherent sheaf [22, p. 101] of positive rank over a surface $S$ with trivial canonical bundle, then $\operatorname{Tr}^{1}$ is surjective, $\operatorname{Tr}^{2}$ is injective and therefore the natural transformation $\left.\operatorname{Def}_{(X, \mathcal{F})} \rightarrow \operatorname{Def}_{(X, \operatorname{det}} \mathcal{F}\right)$ is smooth. When $\mathcal{F}$ is locally free, Theorem 1.2 was already proved in [13] by using transcendental methods, and hence over the field of complex numbers. 
Our proof of Theorem 1.2 is almost entirely algebraic and it relies on the the explicit description of a DG-Lie algebra controlling the deformations of the pair $(X, \mathcal{F})$ and the extension of the trace maps.

Another derived extension of the determinant map was introduced in [24] from the derived stack of perfect complex to derived stack of line bundle.

The paper goes as follows. The first sections of this paper are devoted to the introduction and the study of the first properties of the module of derivations of pairs; we prove that it behaves very well with respect to all the canonical constructions, injective and projective resolutions, de Rham complexes and Fitting stratifications. In Section 5, we define a coherent sheaf of DG-Lie algebras associated with derivations of pairs and the extension of the trace map (Theorem 5.6), that generalises the classical one given in (1.1). Section 6 is included for readers's convenience: here we review the relevant notions on deformation functors associated with a differential graded Lie algebras and with a semicosimplicial DGLie algebras. In Section 7, we analyse the infinitesimal deformations of pairs, describing the DG-Lie algebra that controls these deformations (Theorem 7.11) and we prove the main theorem (Theorem 1.2) about the trace map (Theorem 7.16).

\section{DERIVATIONS AND AUTOMORPHiSMS OF PAIRS}

Let $A \rightarrow R$ be a morphism of unitary commutative rings and $M, N$ two $R$-modules. We shall denote by $\operatorname{Hom}_{A}(M, N)$ (resp.: $\operatorname{Hom}_{R}(M, N)$ ) the $R$-module of $A$-linear (resp.: $R$-linear) maps $M \rightarrow N$ : the $R$-module structure on $\operatorname{Hom}_{A}(M, N)$ is induced by the $R$ module structure on $N$. We shall denote by $\operatorname{Der}_{A}(R, N)=\operatorname{Hom}_{R}\left(\Omega_{R / A}, N\right)$ the $R$-module of $A$-linear derivations $R \rightarrow N$. We shall refer to the $R$-module $R \oplus M$ as the trivial extension whenever $R \oplus M$ is considered as a commutative ring equipped with the product $(r, m)(s, n)=(r s, r n+s m)$, see e.g., [25, p.10].

Definition 2.1. Let $A \rightarrow R$ be a morphism of unitary commutative rings and $M$ an $R$-module. The $R$-module of $A$-derivations of the pair $(R, M)$ is defined as

$$
D_{A}(R, M)=\left\{\begin{array}{l|l}
(h, u) \in \operatorname{Der}_{A}(R, R) \times \operatorname{Hom}_{A}(M, M) & \begin{array}{l}
u(r m)-r u(m)=h(r) m, \\
\text { for every } r \in R, m \in M
\end{array}
\end{array}\right\} .
$$

Remark 2.2. In the setup of the above definition:

(1) if $r_{1}, r_{2}, \ldots \in R$ generate $R$ as an $A$-algebra and $m_{1}, m_{2}, \ldots \in M$ generate $M$ as $R$-module, then every $(h, u) \in D_{A}(R, M)$ is uniquely determined by $h\left(r_{1}\right), h\left(r_{2}\right), \ldots$ and $u\left(m_{1}\right), u\left(m_{2}\right), \ldots$;

(2) if $R \oplus M$ is the trivial extension of $R$ by $M$, then a pair $(h, u) \in \operatorname{Hom}_{A}(R, R) \times$ $\operatorname{Hom}_{A}(M, M)$ belongs to $D_{A}(R, M)$ if and only if the map $R \oplus M \rightarrow R \oplus M$, $(r, m) \mapsto(h(r), u(m))$ is an $A$-derivation.

Lemma 2.3. There exists an exact sequence of $R$-modules

$$
0 \rightarrow D_{A}(R, M) \rightarrow \operatorname{Der}_{A}(R \oplus M, R \oplus M) \stackrel{\Phi}{\rightarrow} \operatorname{Hom}_{R}(M, R) \oplus \operatorname{Der}_{A}(R, M),
$$

where the $R$-module structure on the derivations of the trivial extension $R \oplus M$ is induced by the inclusion $R \rightarrow R \oplus M$.

Proof. Every element of $\operatorname{Hom}_{A}(R \oplus M, R \oplus M)$ is represented by a matrix of $A$-linear maps $\left(\begin{array}{ll}a & b \\ c & d\end{array}\right)$, with

$$
a \in \operatorname{Hom}_{A}(R, R), \quad b \in \operatorname{Hom}_{A}(M, R), \quad c \in \operatorname{Hom}_{A}(R, M), \quad d \in \operatorname{Hom}_{A}(M, M),
$$

and then there exists a natural isomorphism of $R$-modules

$$
\operatorname{Hom}_{A}(R \oplus M, R \oplus M)=\operatorname{Hom}_{A}(R, R) \oplus \operatorname{Hom}_{A}(M, R) \oplus \operatorname{Hom}_{A}(R, M) \oplus \operatorname{Hom}_{A}(M, M) \text {. }
$$


We have already noticed that $(h, u) \in D_{A}(R, M)$ if and only if $\left(\begin{array}{cc}h & 0 \\ 0 & u\end{array}\right) \in \operatorname{Der}_{A}(R \oplus M, R \oplus$ $M)$. Therefore, defining $\Phi\left(\begin{array}{ll}a & b \\ c & d\end{array}\right)=(b, c)$, it is straightforward to see that if $\left(\begin{array}{ll}a & b \\ c & d\end{array}\right)$ is a derivation, then $b$ is $R$-linear and $c$ is a derivation. It also easy to prove that the image of $\Phi$ is

$$
\left\{b \in \operatorname{Hom}_{R}(M, R) \mid b(m) n+m b(n)=0 \quad \forall m, n \in M\right\} \oplus \operatorname{Der}_{A}(R, M) .
$$

Lemma 2.4. In the setup of Definition 2.1, let us denote by

$$
q: D_{A}(R, M) \rightarrow \operatorname{Hom}_{A}(M, M) \quad \text { and } \quad \alpha: D_{A}(R, M) \rightarrow \operatorname{Der}_{A}(R, R),
$$

the projection maps restricted to $D_{A}(R, M)$ :

(1) if $M$ is a faithful module, i.e., if ann $(M)=0$, then $q$ is injective and its image is the submodule of differential operators of first order with principal symbol;

(2) there exists an exact sequence of $R$-modules

$$
0 \rightarrow \operatorname{Hom}_{R}(M, M) \rightarrow D_{A}(R, M) \stackrel{\alpha}{\longrightarrow} \operatorname{Der}_{A}(R, R) .
$$

Proof. The only nontrivial statement is the one concerning the image of $q$. Recall that a morphism $v \in \operatorname{Hom}_{A}(M, M)$ is a differential operator of first order if for every $r \in R$ the map

$$
[v, r]: M \rightarrow M, \quad[v, r](m)=v(r m)-r v(m),
$$

is a morphism of $R$-modules. In this case the symbol $\sigma(v)$ is defined as the map

$$
\sigma(v): R \rightarrow \operatorname{Hom}_{R}(M, M), \quad \sigma(v)(r)=[v, r],
$$

and it is called principal if $\sigma(v)(r)$ is a scalar multiple of the identity for every $r \in R$. Notice that the symbol $\sigma(v)$ is an $A$-derivation, since for every $r, s \in R$ we have

$$
\sigma(v)(r s)=[v, r s]=[v, r] s+r[v, s] .
$$

If $(h, u) \in D_{A}(R, M)$, then for every $r \in R$

$$
[u, r]=h(r) \operatorname{Id}_{M} \in \operatorname{Hom}_{R}(M, M) .
$$

Conversely, let $v \in \operatorname{Hom}_{A}(M, M)$ be a first order differential operator such that $\sigma(v)(R) \subseteq$ $R \operatorname{Id}_{M}$. Since $M$ is faithful, for every $r \in R$ there exists a unique $h(r) \in R$ such that $\sigma(v)(r)=[v, r]=h(r) \operatorname{Id}_{M}$. Since $\sigma(v)$ is an $A$-derivation, also $h: R \rightarrow R$ is an $A$-derivation and therefore $(h, v) \in D_{A}(R, M)$.

The restriction $\alpha: D_{A}(R, M) \rightarrow \operatorname{Der}_{A}(R, R)$ of the projection on the first factor is called the anchor map of the pair. It is plain that $D_{A}(R, M)$ is a Lie subalgebra of $\operatorname{Der}_{A}(R, R) \times$ $\operatorname{Hom}_{A}(M, M)$ and we have the relation

$$
[x, r y]=\alpha(x)(r) y+r[x, y], \quad x, y \in D_{A}(R, M), \quad r \in R,
$$

called Poisson identity. Keep attention to the fact that the bracket on $\operatorname{Der}_{A}(R, R)$, and then on $D_{A}(R, M)$, is bilinear over $A$ and not over $R$.

Example 2.5 (Lie derivative). Let $A \rightarrow R$ be a morphism of unitary commutative rings. Then, every $h \in \operatorname{Der}_{A}(R, R)$ gives a canonical element $\left(h, L_{h}\right) \in D_{A}\left(R, \Omega_{R / A}\right)$ uniquely determined by the equation $L_{h}(d x)=d(h(x)), x \in R$.

Recall that we may define the module of Kähler differentials as $\Omega_{R / A}=I / I^{2}$, where $I$ is the kernel of the multiplication map $R \otimes_{A} R \rightarrow R$, the differential $d x$ is the class of $x \otimes 1-1 \otimes x$ in $I / I^{2}$ and the $R$-module structure is induced by the morphism of $A$-algebras 
$R \rightarrow R \otimes_{A} R, r \mapsto r \otimes 1$, see e.g., [21, Section 25]. For every $h \in \operatorname{Der}_{A}(R, R)$ we define $L_{h}: I / I^{2} \rightarrow I / I^{2}$ as the factorization to the quotient of the derivation

$$
k: R \otimes_{A} R \rightarrow R \otimes_{A} R, \quad k(x \otimes y)=h(x) \otimes y+x \otimes h(y) .
$$

The equation $L_{h}(d x)=d(h(x))$ is trivially satisfied. For every $r, s, x \in R$, we have

$$
\begin{aligned}
L_{h}(r s d x) & =k((r \otimes 1)(s x \otimes 1-s \otimes x)) \\
& =(h(r) \otimes 1)(s x \otimes 1-s \otimes x)+(r \otimes 1) k(s x \otimes 1-s \otimes x)=h(r) s d x+r L_{h}(s d x),
\end{aligned}
$$

and then $\left(h, L_{h}\right) \in D_{A}\left(R, \Omega_{R / A}\right)$.

The definition of $D_{A}(R, M)$ extends naturally to $D_{A}\left(R, M_{\bullet}\right)$, where $M_{\bullet}$ is a diagram of $R$-modules over a small category $I$. Here we are mainly interested in two cases. The former is when $I$ is just a set, a diagram of $R$-modules is just a collection $\left\{M_{i}\right\}, i \in I$, and $D_{A}\left(R, M_{\bullet}\right)$ is the limit of the diagram of anchor maps $\alpha: D_{A}\left(R, M_{i}\right) \rightarrow \operatorname{Der}_{A}(R, R)$. For later use, it is notational convenient to denote

$$
\begin{aligned}
\prod_{i}^{\times} D_{A}\left(R, M_{i}\right) & =D_{A}\left(R, M_{\bullet}\right)=\lim _{i}\left(\alpha: D_{A}\left(R, M_{i}\right) \rightarrow \operatorname{Der}_{A}(R, R)\right) \\
& =\left\{\left(h, \ldots, u_{i}, \ldots\right) \mid\left(h, u_{i}\right) \in D_{A}\left(R, M_{i}\right), \quad \forall i \in I\right\} .
\end{aligned}
$$

The latter is when $M_{\bullet}=\left\{M_{1} \stackrel{f}{\rightarrow} M_{2}\right\}$ is a morphism of two $R$-modules, then $D_{A}\left(R, M_{\bullet}\right)$ is the set of triples $\left(h, u_{1}, u_{2}\right)$, with $\left(h, u_{1}\right) \in D_{A}\left(R, M_{1}\right),\left(h, u_{2}\right) \in D_{A}\left(R, M_{2}\right)$, and $f u_{1}=$ $u_{2} f$. Therefore, there exists an exact sequence of $R$-modules

$$
0 \rightarrow D_{A}\left(R, M_{\bullet}\right) \rightarrow D_{A}\left(R, M_{1}\right) \times_{\operatorname{Der}_{A}(R, R)} D_{A}\left(R, M_{2}\right) \stackrel{\Phi}{\longrightarrow} \operatorname{Hom}_{R}\left(M_{1}, M_{2}\right),
$$

where

$$
\Phi\left(\left(h, u_{1}\right),\left(h, u_{2}\right)\right)=f u_{1}-u_{2} f .
$$

Lemma 2.6. Let $A \rightarrow R$ be a morphism of unitary commutative rings and $M, N$ two $R$-modules. Then, we have two natural R-linear morphisms of Lie algebras:

$$
\begin{aligned}
D_{A}\left(R, M \otimes_{R} N\right) \stackrel{\Phi}{\leftarrow} D_{A}(R, M) \times_{\operatorname{Der}_{A}(R, R)} D_{A}(R, N) \stackrel{\Psi}{\rightarrow} D_{A}\left(R, \operatorname{Hom}_{R}(M, N)\right), \\
\Phi((h, u),(h, v))=\left(h, u \otimes_{R} \operatorname{Id}_{N}+\operatorname{Id}_{M} \otimes_{R} v\right), \\
\Psi((h, u),(h, v))=(h, f \mapsto v f-f u) .
\end{aligned}
$$

Proof. Straightforward. Notice that $u \otimes_{R} \operatorname{Id}_{N}+\operatorname{Id}_{M} \otimes_{R} v$ is well defined, although $u, v$ are not $R$-linear and the two addends $u \otimes_{R} \operatorname{Id}_{N}, \operatorname{Id}_{M} \otimes_{R} v$ are not defined. Similarly $v f-f u$ is a morphism of $R$-modules, although $v f$ and $f u$ are only $A$-linear.

As a particular case of Lemma 2.6 we obtain a natural $R$-linear morphisms of Lie algebras (the transpose)

$$
\begin{aligned}
(-)^{T}: D_{A}(R, M) & \rightarrow D_{A}\left(R, \operatorname{Hom}_{R}(M, R)\right), \\
(h, u) & \mapsto(h, u)^{T}=\Psi((h, u),(h, h))=(h, f \mapsto h f-f u) .
\end{aligned}
$$

Definition 2.7. The Leibniz extension of a derivation of pair $(h, u) \in D_{A}(R, M)$ is the sequence $\left(h, u_{n}\right) \in D_{A}\left(R, \wedge_{R}^{n} M\right), n \geq 0$, uniquely determined by the formulas:

$$
u_{0}=h, \quad u_{n}\left(m_{1} \wedge \cdots \wedge m_{n}\right)=\sum_{i=1}^{n} m_{1} \wedge \cdots \wedge u\left(m_{i}\right) \wedge \cdots \wedge m_{n} .
$$


The Leibniz extension is properly defined: by the universal property of wedge products, the Formula (2.5) defines a sequence of $A$-linear maps

$$
\tilde{u}_{n}: \wedge_{A}^{n} M \rightarrow \wedge_{A}^{n} M .
$$

Now $\wedge_{R}^{n} M$ is the quotient of $\wedge_{A}^{n} M$ by the $A$-submodule $H$ generated by all the elements $m_{1} \wedge \cdots \wedge r m_{i} \wedge m_{i+1} \wedge \cdots \wedge m_{n}-m_{1} \wedge \cdots \wedge m_{i} \wedge r m_{i+1} \wedge \cdots \wedge m_{n}, \quad r \in R, m_{j} \in M, 0<i<n$, and it is immediate to verify that $\tilde{u}_{n}(H) \subset H$ and then that $\left(h, \tilde{u}_{n}\right)$ factors to a derivation of pair $\left(h, u_{n}\right) \in D_{A}\left(R, \wedge_{R}^{n} M\right)$. The Leibniz extension is functorial in the following sense: given a morphism of $R$-modules $f: M \rightarrow N$ and $(h, u) \in D_{A}(R, M),(h, v) \in D_{A}(R, N)$ such that $v f=f u$, then $v_{n} f^{\wedge n}=f^{\wedge n} u_{n}$ for every $n$. Moreover, for every $n$, the map

$$
D_{A}(R, M) \rightarrow D_{A}\left(R, \wedge_{R}^{n} M\right), \quad(h, u) \mapsto\left(h, u_{n}\right),
$$

is a morphism of Lie algebras. This follows immediately from the fact that, for every $(h, u),(k, v) \in D_{A}(R, M)$ we have

$$
\begin{aligned}
u_{n} v_{n}\left(m_{1} \wedge \cdots \wedge m_{n}\right)= & \sum_{i=1}^{n} m_{1} \wedge \cdots \wedge u v\left(m_{i}\right) \wedge \cdots \wedge m_{n} \\
& +\sum_{i<j} m_{1} \wedge \cdots u\left(m_{i}\right) \wedge \cdots \wedge v\left(m_{j}\right) \cdots \wedge m_{n} \\
& +\sum_{i<j} m_{1} \wedge \cdots v\left(m_{i}\right) \wedge \cdots \wedge u\left(m_{j}\right) \cdots \wedge m_{n} .
\end{aligned}
$$

Definition 2.8. If $M$ is a free module of rank $n$ and $(h, u) \in D_{A}(R, M)$, we shall call $\left(h, u_{n}\right) \in D_{A}\left(R, \wedge_{R}^{n} M\right)$ the trace of $(h, u)$.

The name trace is motivated by the fact that if $h=0$, i.e., if $u \in \operatorname{Hom}_{R}(M, M)$, then $u_{n}$ is the multiplication by the trace of $u$.

Automorphisms of pairs. Let $A \rightarrow R$ be a morphism of commutative unitary rings and let $M$ be an $R$-module. We shall denote by $\operatorname{Aut}_{A}(R)$ the group of $A$-linear automorphisms of $R$, i.e., the automorphism group of the $A$-algebra $R$, and by $\operatorname{Aut}_{A}(M)\left(\operatorname{resp}: \operatorname{Aut}_{R}(M)\right)$ the group of $A$-linear (resp.: $R$-linear) automorphisms of $M$.

Definition 2.9. The group of $A$-linear automorphisms of the pair $(R, M)$ is defined as the subgroup $\operatorname{Aut}_{A}(R, M) \subset \operatorname{Aut}_{A}(R) \times \operatorname{Aut}_{A}(M)$ of pairs $(\theta, \phi)$ such that $\phi(r m)=\theta(r) \phi(m)$ for every $r \in R, m \in M$.

Let $R \oplus M$ be the trivial extension of $R$ by $M$. As in the proof of Lemma 2.3, there exists a natural inclusion

$$
\operatorname{Hom}_{A}(R, R) \times \operatorname{Hom}_{A}(M, M) \subset \operatorname{Hom}_{A}(R \oplus M, R \oplus M)
$$

and it is immediate to see that an element of $\operatorname{Hom}_{A}(R, R) \times \operatorname{Hom}_{A}(M, M)$ is an automorphism of the pair $(R, M)$ if and only if it is an automorphism of the $A$-algebra $R \oplus M$.

The analog of the anchor map is the group homomorphism

$$
\alpha: \operatorname{Aut}_{A}(R, M) \rightarrow \operatorname{Aut}_{A}(R), \quad \alpha(\theta, \phi)=\theta,
$$

whose kernel is $\operatorname{Aut}_{R}(M)$.

There exists the analog of Lemma 2.6 and of the Leibniz extension for automorphisms of pairs. It is straightforward to verify that there exist two natural group homomorphisms

$$
\operatorname{Aut}_{A}\left(R, M \otimes_{R} N\right) \stackrel{\Phi}{\leftarrow} \operatorname{Aut}_{A}(R, M) \times_{\operatorname{Aut}_{A}(R)} \operatorname{Aut}_{A}(R, N) \stackrel{\Psi}{\rightarrow} \operatorname{Aut}_{A}\left(R, \operatorname{Hom}_{R}(M, N)\right),
$$




$$
\begin{aligned}
& \Phi((\theta, \phi),(\theta, \psi))=(\theta, \phi \otimes \psi), \\
& \Psi((\theta, \phi),(\theta, \psi))=\left(\theta, f \mapsto \psi f \phi^{-1}\right) .
\end{aligned}
$$

Similarly, every $(\theta, \phi) \in \operatorname{Aut}_{A}(R, M)$ gives a sequence $\left(\theta, \phi_{n}\right) \in \operatorname{Aut}_{A}\left(R, \wedge_{R}^{n} M\right), n \geq 0$, uniquely determined by the formulas:

$$
\phi_{0}=\theta, \quad \phi_{n}\left(m_{1} \wedge \cdots \wedge m_{n}\right)=\phi\left(m_{1}\right) \wedge \cdots \wedge \phi\left(m_{n}\right) .
$$

If $M$ is free of rank $n$, we write $\left(\theta, \phi_{n}\right)=\operatorname{det}(\theta, \phi)$ : when $\theta=$ Id we recover the usual notion of the determinant of a $R$-linear endomorphism.

Assume now that $A$ contains the field $\mathbb{Q}$ of rational numbers and let $(h, u) \in D_{A}(R, M) \subset$ $\operatorname{Der}_{A}(R \oplus M, R \oplus M)$ be a nilpotent derivation of pairs. Then, also its exponential

$$
\exp (h, u)=\left(\sum_{n=0}^{\infty} \frac{h^{n}}{n !}, \sum_{n=0}^{\infty} \frac{u^{n}}{n !}\right)
$$

preserves the direct sum decomposition $R \oplus M$ and then

$$
\exp (h, u) \in \operatorname{Aut}_{A}(R, M) \subset \operatorname{Aut}_{A}(R \oplus M) .
$$

It is plain that the exponential commutes with the anchor maps and the usual properties of the exponential imply that exp commutes with the morphisms (2.3) and (2.6). The exponential also commutes with Leibniz extensions of derivations and automorphisms: this is immediate from the previous remark since the assumption $\mathbb{Q} \subset A$ implies that $\bigwedge_{R}^{n} M$ is a direct summand of $\otimes_{R}^{n} M$. In particular, the exponential of the trace is the determinant of the exponential.

Lemma 2.10. Let $R$ be a commutative unitary algebra over a field $\mathbb{K}$ of characteristic 0 , and let $M$ be an $R$-module. Then, for every local Artin $\mathbb{K}$-algebra $A$ with residue field $\mathbb{K}$, the group $\exp \left(D_{\mathbb{K}}(R, M) \otimes \mathfrak{m}_{A}\right)$ is naturally isomorphic to the group of $A$-linear automorphisms of the pair $(R \otimes A, M \otimes A)$ lifting the identity on $(R, M)$.

Proof. We have already noticed that via the diagonal inclusion

$$
\operatorname{Hom}_{\mathbb{K}}(R, R) \times \operatorname{Hom}_{\mathbb{K}}(M, M) \subset \operatorname{Hom}_{\mathbb{K}}(R \oplus M, R \oplus M),
$$

a couple $(h, u) \in \operatorname{Hom}_{\mathbb{K}}(R, R) \times \operatorname{Hom}_{\mathbb{K}}(M, M)$ is a $\mathbb{K}$-linear derivation (resp.: automorphism) of the pair $(R, M)$ if and only if it is a $\mathbb{K}$-linear derivation (resp.: automorphism) of the trivial extension $R \oplus M$. Thus, the lemma is an immediate consequence of the well known fact (see e.g. [17, Proposition 5.44]) that for every commutative unitary $\mathbb{K}$-algebra $S$, the group $\exp \left(\operatorname{Der}_{\mathbb{K}}(S, S) \otimes \mathfrak{m}_{A}\right)$ is naturally isomorphic to the group of $A$-linear automorphisms of $S \otimes A$ lifting the identity on $S$.

\section{LifTING TO RESOLUTIONS}

Throughout this section, $A \rightarrow R$ is a fixed morphism of unitary commutative rings. For every $(h, u) \in D_{A}(R, M)$ the derivation $h$ preserves the annihilator of $M$ : in fact, if $r \in R$ and $r m=0$ for every $m \in M$, then also $h(r) m=u(r m)-r u(m)=0$, for every $m \in M$.

The above consideration shows that in general the anchor map $\alpha: D_{A}(R, M) \rightarrow \operatorname{Der}_{A}(R, R)$ is not surjective. For instance, if $M=R / I$, with $I$ an ideal of $R$, then the image of $\alpha$ is the submodule of derivations preserving the ideal $I$ : if $(h, u) \in D_{A}(R, M)$ we have seen that $h$ preserves the annihilator of $M$, which is precisely the ideal $I$. Conversely, any derivation $h \in \operatorname{Der}_{A}(R, R)$ has a canonical lifting $(h, h) \in D_{A}(R, R)$, and if $h(I) \subset I$, then $(h, h)$ factors to an element of $D_{A}(R, M)$.

Lemma 3.1. In the above setup, if $f: P \rightarrow M$ is a surjective morphism of $R$-modules with $P$ projective, then every $(h, u) \in D_{A}(R, M)$ lifts to an element $(h, v) \in D_{A}(R, P)$ such that $f v=u f$. In particular: 
(1) every derivation of pairs lifts to any projective resolution;

(2) if $P$ is a projective $R$-module, then the anchor map $\alpha: D_{A}(R, P) \rightarrow \operatorname{Der}_{A}(R, R)$ is surjective.

Proof. Consider first the case when $P$ is a free $R$-module with basis $\left\{e_{i}\right\}$. Choosing elements $v_{i} \in P$ such that $f\left(v_{i}\right)=u\left(f\left(e_{i}\right)\right) \in M$ for every $i$, then the $A$-linear map

$$
v: P \rightarrow P, \quad v\left(\sum_{i} a_{i} e_{i}\right)=\sum_{i} a_{i} v_{i}+h\left(a_{i}\right) e_{i},
$$

has the required properties. If $P$ is not free, since every projective module is a direct summand of a free module, there exist a free module $F$ together with two morphisms $i: P \rightarrow F, g: F \rightarrow P$ such that $g i=\operatorname{Id}_{P}$. Since $f g: F \rightarrow M$ is surjective there exists $(h, w) \in D_{A}(R, F)$ lifting $(h, u)$ and it is sufficient to take $(h, v)=(h, g w i) \in D_{A}(R, P)$. If $M=0$ then for every $h \in \operatorname{Der}_{A}(R, R)$ we have $(h, 0) \in D_{A}(R, 0)$ and the above computation gives the surjectivity of the anchor map $\alpha: D_{A}(R, P) \rightarrow \operatorname{Der}_{A}(R, R)$.

Lemma 3.2. In the above setup, if $g: M \rightarrow J$ is an injective morphism of $R$-modules with $J$ injective, then every $(h, u) \in D_{A}(R, M)$ extends to an element $(h, v) \in D_{A}(R, J)$ such that $v g=g u$. In particular:

(1) every derivation of pairs extends to any injective resolution;

(2) if $J$ is a injective $R$-module, then the anchor map $\alpha: D_{A}(R, J) \rightarrow \operatorname{Der}_{A}(R, R)$ is surjective.

Proof. Let $q: J \rightarrow I$ be an injective morphism with $I$ injective as an $A$-module, then the composition $q g: M \rightarrow J \rightarrow I$ is also injective. Consider the $R$-module $\operatorname{Hom}_{A}(R, I)$, where the $R$-module structure is given by $(t \psi)(r)=\psi(t r)$, for any $t, r \in R$ and any $\psi \in$ $\operatorname{Hom}_{A}(R, I)$. Then, the map

$$
\beta: J \rightarrow \operatorname{Hom}_{A}(R, I) \quad j \mapsto \beta(j)(r)=q(r j) \in I, \quad \forall r \in R,
$$

is an injective $R$-linear morphism. Since $J$ is injective as $R$-module, there exists a splitting $\gamma: \operatorname{Hom}_{A}(R, I) \rightarrow J$, such that $\gamma \beta=\operatorname{Id}_{J}$. Let $(h, u) \in D_{A}(R, M)$ be a fixed derivation of the pair; since $I$ is injective as $A$-module and $u$ is a morphism of $A$-modules, there exists a morphism $w \in \operatorname{Hom}_{A}(I, I)$ such that $w q g=q g u$ :

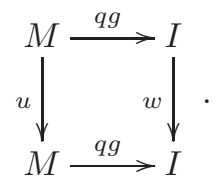

Consider now the map $w_{h}: \operatorname{Hom}_{A}(R, I) \rightarrow \operatorname{Hom}_{A}(R, I)$ defined by $w_{h}(\psi)=w \circ \psi-\psi \circ h$, for any $\psi \in \operatorname{Hom}_{A}(R, I)$. Then, $\left(h, w_{h}\right) \in D_{A}\left(R, \operatorname{Hom}_{A}(R, I)\right)$ since $w_{h}$ is $A$-linear and $w_{h}(t \psi)=t w_{h}(\psi)+h(t) \psi$, for any $t, r \in R$; indeed, we have:

$$
w_{h}(t \psi)(r)=(w \circ t \psi-t \psi \circ h)(r)=w(\psi(t r))-\psi(t h(r)),
$$

while

$t w_{h}(\psi)(r)=t(w \circ \psi-\psi \circ h)(r)=w(\psi(t r))-\psi(h(t r))=w(\psi(t r))-\psi(t h(r))-\psi(r h(t))$.

Then, we prove that $\left(h, w_{h}\right)$ extends $(h, u)$, i.e., $\beta g u=w_{h} \beta g$. For every $r \in R$ and every $m \in M$, we have

$$
\begin{aligned}
\left(w_{h} \beta g(m)\right)(r) & =w(\beta g(m)(r))-\beta g(m)(h(r))=w(q(r g(m))-q(h(r) g(m)) \\
& =w(q(g(r m))-q(g(h(r) m))=q g u(r m)-q(g(h(r) m)) \\
& =q g(r u(m))+q g(h(r) m)-q(g(h(r) m))=q g(r u(m))=\beta g(u(m))(r) .
\end{aligned}
$$


Finally, it is sufficient to take $(h, v)=\left(h, \gamma w_{h} \beta\right) \in D_{A}(R, J)$. Item (1) is now clear and item (2) follows by considering the injective morphism $0 \rightarrow J$.

Corollary 3.3. Let $0 \rightarrow K \stackrel{\alpha}{\rightarrow} P \stackrel{\beta}{\rightarrow} M \rightarrow 0$ be a short exact sequence of projective $R$-modules, and denote by

$$
L=D_{A}(R, K \stackrel{\alpha}{\rightarrow} P)=D_{A}(R, P \stackrel{\beta}{\rightarrow} M)=\left\{(h, u) \in D_{A}(R, P) \mid u(\alpha(K)) \subset \alpha(K)\right\} .
$$

Then, the natural morphisms of Lie algebras

$$
\begin{gathered}
L=D_{A}(R, K \stackrel{\alpha}{\rightarrow} P) \rightarrow D_{A}(R, K), \quad L=D_{A}(R, K \stackrel{\alpha}{\rightarrow} P) \rightarrow D_{A}(R, P), \\
D_{A}(R, P \stackrel{\beta}{\rightarrow} M) \rightarrow D_{A}(R, M),
\end{gathered}
$$

fit in the following exact sequences of $R$-modules

$$
\begin{aligned}
& 0 \rightarrow L \rightarrow D_{A}(R, P) \stackrel{p}{\rightarrow} \operatorname{Hom}_{R}(K, M) \rightarrow 0, \quad 0 \rightarrow \operatorname{Hom}_{R}(P, K) \stackrel{j}{\rightarrow} L \rightarrow D_{A}(R, M) \rightarrow 0, \\
& \qquad 0 \rightarrow \operatorname{Hom}_{R}(M, P) \stackrel{h}{\rightarrow} L \rightarrow D_{A}(R, K) \rightarrow 0, \\
& \text { where } p(h, u)=\beta u \alpha, j(v)=(0, \alpha v), h(u)=(0, u \beta) .
\end{aligned}
$$

Proof. Since $\beta \alpha=0$, the morphism $p$ is properly defined and it is surjective because $M$ is projective, $P \simeq K \oplus M$ and therefore

$$
\operatorname{Hom}_{R}(P, P) \rightarrow \operatorname{Hom}_{R}(K, M), \quad u \mapsto \beta u \alpha,
$$

is surjective. The surjectivity of $L \rightarrow D_{A}(R, M)$ is given by Lemma 3.1 and therefore also the anchor map $\alpha: L \rightarrow \operatorname{Der}_{A}(R, R)$ is surjective. The third exact sequence follows by the snake lemma applied to the commutative diagram

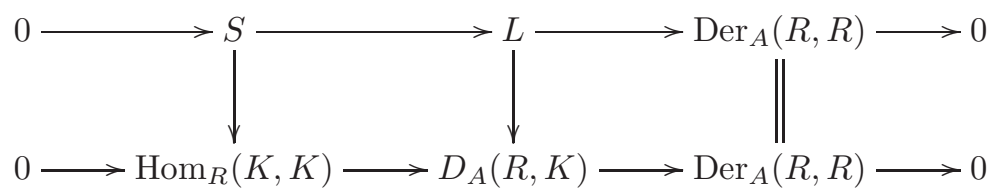

where $S=\left\{f \in \operatorname{Hom}_{R}(P, P) \mid f(K) \subset K\right\}$.

Consider now a morphism of commutative unitary rings $A \rightarrow R$ and a cochain complex $C=\left\{\cdots \rightarrow C^{i} \stackrel{d}{\rightarrow} C^{i+1} \rightarrow \cdots\right\}$ of $R$ modules. Then, we can define $D_{A}(R, C)$ as in Definition 2.1, by replacing $\operatorname{Hom}_{A}(M, M)$ with the module of morphisms of cochain complexes of $A$-modules; equivalently $D_{A}(R, C)$ is defined considering $C$ as a diagram of $R$-modules over the ordered set $\mathbb{Z}$.

However, for the application we have in mind, it is more convenient to consider the DG-Lie subalgebra

$$
D_{A}^{*}(R, C)=\left\{\begin{array}{l|l}
(h, u) \in \operatorname{Der}_{A}^{*}(R, R) \times \operatorname{Hom}_{A}^{*}(C, C) \mid \begin{array}{l}
u(r x)-r u(x)=h(r) x, \\
\text { for every } r \in R, x \in C
\end{array}
\end{array}\right\} .
$$

For the definition and the main properties of $\operatorname{Der}_{A}^{*}(R, R)$ and $\operatorname{Hom}_{A}^{*}(C, C)$ the reader may consult e.g., [15, Section 1]. Notice that the differential is the internal derivation $\delta=[d,-]$ and $\operatorname{Der}_{A}^{i}(R, R)=0$ for every $i \neq 0$ : this implies that $D_{A}^{i}(R, C)=\operatorname{Hom}_{R}^{i}(C, C)$ for every $i \neq$ 0 , and $D_{A}^{0}(R, C)=\prod^{\times} D_{A}\left(R, C^{i}\right)$ is the limit of the diagram of anchor maps $D_{A}\left(R, C^{i}\right) \rightarrow$ $\operatorname{Der}_{A}(R, R)$. Finally, note that $D_{A}(R, C)=Z^{0}\left(D_{A}^{*}(R, C)\right)$.

In order to extend Lemma 3.1 and Corollary 3.3 to the differential graded case it is useful to work in the projective model structure on the category of unbounded cochain complexes [12, Theorem 2.3.11]: with respect to this model structure, a morphism of cochain complexes $M \rightarrow N$ is a weak-equivalence if it is a quasi-isomorphism, it is a fibration if it is degreewise surjective, it is a cofibration if it has the left lifting property with respect to all the trivial 
fibrations. Moreover, if $M \rightarrow N$ is a cofibration, then for every $i$ the map $M^{i} \rightarrow N^{i}$ is split injective with projective cokernel; the converse holds whenever $N$ is bounded above.

In particular, for every cofibrant complex $P$, since $P^{i}$ is projective for every $i$, there exists a short exact sequence

$$
0 \rightarrow \operatorname{Hom}_{R}^{*}(P, P) \rightarrow D_{A}^{*}(R, P) \rightarrow \operatorname{Der}_{A}(R, R) \rightarrow 0 .
$$

Proposition 3.4. Let $f: K \rightarrow P$ be a trivial cofibration between cofibrant complexes of $R$-modules. Then, the two natural DG-Lie algebra morphisms

$$
D_{A}^{*}(R, K) \longleftarrow D_{A}^{*}(R, K \stackrel{f}{\rightarrow} P) \longrightarrow D_{A}^{*}(R, P)
$$

are quasi-isomorphisms of complexes of $R$-modules. In particular, $D_{A}^{*}(R, K)$ and $D_{A}^{*}(R, P)$ are quasi-isomorphic DG-Lie algebras.

Proof. By assumption $f$ is a cofibration and then we have a short exact sequence of cofibrant complexes $0 \rightarrow K \stackrel{f}{\rightarrow} P \rightarrow M \rightarrow 0$. Since $K^{i}, P^{i}, M^{i}$ are projective modules for every $i$, Corollary 3.3 gives two short exact sequences of complexes

$$
\begin{aligned}
& 0 \rightarrow D_{A}^{*}(R, K \stackrel{f}{\rightarrow} P) \rightarrow D_{A}^{*}(R, P) \rightarrow \operatorname{Hom}_{R}^{*}(K, M) \rightarrow 0, \\
& 0 \rightarrow \operatorname{Hom}_{R}^{*}(M, P) \rightarrow D_{A}^{*}(R, K \stackrel{f}{\rightarrow} P) \rightarrow D_{A}^{*}(R, K) \rightarrow 0 .
\end{aligned}
$$

Finally, since $M$ is cofibrant acyclic, the natural map $M \rightarrow \operatorname{Cone}\left(\operatorname{Id}_{M}\right)$ is a trivial cofibration and then admits a left inverse; hence the complexes $M, \operatorname{Hom}_{R}^{*}(M, P)$ and $\operatorname{Hom}_{R}^{*}(K, M)$ are contractible.

Corollary 3.5. Let $P$ and $K$ be quasi-isomorphic cofibrant complexes of $R$-modules. Then, $D_{A}^{*}(R, P)$ is quasi-isomorphic to $D_{A}^{*}(R, K)$ as a $D G$-Lie algebra.

Proof. By general facts of model category theory, see e.g. [12, Lemma 1.1.12], two cofibrant complexes $P$ and $K$ are quasi-isomorphic if and only if there exists a span of trivial cofibrations $P \rightarrow Q \leftarrow K$. Therefore, the conclusion follows from Proposition 3.4.

Example 3.6. If $P \rightarrow M$ is a projective resolution of an $R$-module $M$, then Corollary 3.5 implies that the graded Lie algebra $H^{*}\left(D_{A}^{*}(R, P)\right)$ depends only on $M$. Moreover, by (3.1) we have $H^{i}\left(D_{A}^{*}(R, P)\right)=\operatorname{Ext}_{R}^{i}(M, M)$ for every $i \neq 0,1$ and, by (2.1) of Lemma 2.4, there exists an exact sequence

$0 \rightarrow \operatorname{Ext}_{R}^{0}(M, M) \rightarrow H^{0}\left(D_{A}^{*}(R, P)\right) \rightarrow \operatorname{Der}_{A}(R, R) \rightarrow \operatorname{Ext}_{R}^{1}(M, M) \rightarrow H^{1}\left(D_{A}^{*}(R, P)\right) \rightarrow 0$.

In fact $\operatorname{Ext}_{R}^{i}(M, M)=H^{i}\left(\operatorname{Hom}_{R}^{*}(P, M)\right)=H^{i}\left(\operatorname{Hom}_{R}^{*}(P, P)\right)$ for every $i$. By Lemma 3.1, the natural map $Z^{0}\left(D_{A}^{*}(R, P)\right) \rightarrow D_{A}(R, M)$ is surjective; its kernel is given by the $R$-linear morphisms of complexes $P \rightarrow P$ inducing the trivial map on $M$. Since $P$ is a projective resolution, these morphisms are exactly the ones homotopic to 0 , and then there exists a natural isomorphism $H^{0}\left(D_{A}^{*}(R, P)\right)=D_{A}(R, M)$. Since $\operatorname{Ext}_{R}^{i}(M, M)=0$ for $i<0$, the above equality is completely equivalent to the long exact sequence

$$
\cdots \rightarrow \operatorname{Hom}_{R}^{-2}(P, P) \stackrel{\delta}{\rightarrow} \operatorname{Hom}_{R}^{-1}(P, P) \stackrel{\delta}{\rightarrow} D_{A}(R, P) \rightarrow D_{A}(R, M) \rightarrow 0 .
$$




\section{Anchor invariance of Fitting ideals}

The content of this section is not relevant for the remaining part of the paper and it is written as an application of the previous results that we consider of independent interest.

Let $A \rightarrow R$ be a morphism of unitary commutative rings and $M$ an $R$-module. We have seen that, unless $M$ is either projective or injective, the anchor map $D_{A}(R, M) \stackrel{\alpha}{\longrightarrow}$ $\operatorname{Der}_{A}(R, R)$ is generally not surjective.

Theorem 4.1. In the above setup, if $M$ is finitely generated, then for every $(h, u) \in$ $D_{A}(R, M)$ the derivation $h$ preserves the Fitting ideals of $M$.

Proof. Recall (see $[4,26])$ that the Fitting ideals of $M, \operatorname{Fitt}_{0}(M) \subset \operatorname{Fitt}_{1}(M) \subset \cdots \subset R$, are defined by considering any free resolution

$$
F \stackrel{f}{\rightarrow} R^{m} \stackrel{p}{\longrightarrow} M \rightarrow 0
$$

and the exterior powers $\wedge_{R}^{i} F \stackrel{f^{\wedge i}}{\longrightarrow} \wedge_{R}^{i} R^{m}$. Then, for every $i \geq 0$, the Fitting ideal Fitt $_{m-i}(M) \subset R$ is the ideal generated by the coefficients of all elements in the image of $f^{\wedge i}$, with respect to the canonical basis of $\wedge_{R}^{i} R^{m}$. The definition is independent of the choice of the resolution and then $\operatorname{Fitt}_{m}(M)=R$ whenever $M$ is generated by $m$ elements.

By Lemma 3.1, the derivation of pair $(h, u)$ lifts to a couple $(h, v) \in D_{A}\left(R, R^{m}\right)$ and $(h, w) \in D_{A}(R, F)$ such that $u p=p v$ and $f w=v f$. Let us prove first that the ideal $I=\operatorname{Fitt}_{m-1}(M)$ is preserved by $h$. Let $e_{1}, \ldots, e_{m}$ be the canonical basis of $R^{m}$. For every $x \in F$, we have

$$
f(x)=\sum a_{i} e_{i}, \quad f(w(x))=\sum b_{i} e_{i}, \quad a_{i}, b_{i} \in I
$$

and then

$$
\sum h\left(a_{i}\right) e_{i}=v(f(x))-\sum a_{i} v\left(e_{i}\right)=\sum b_{i} e_{i}-\sum a_{i} v\left(e_{i}\right) \in I \cdot R^{m},
$$

proving that $h\left(a_{i}\right) \in I$ for every $i$. As regard the invariance of the Fitting ideals Fitt $m-r(M)$ for $r>1$, it is sufficient to repeat the above argument to the maps $f^{\wedge r}: \wedge_{R}^{r} F \rightarrow \wedge_{R}^{r} R^{m}$ and to the Leibniz extensions of $(h, v)$ and $(h, w)$.

Corollary 4.2. Let $A \rightarrow R$ be a morphism of noetherian rings and let $M$ and $N$ be two finitely generated $R$-modules. If a derivation $h \in \operatorname{Der}_{A}(R, R)$ lifts to $D_{A}(R, M)$ and to $D_{A}(R, N)$, then $h$ preserves the Fitting ideals of $\operatorname{Tor}_{i}^{R}(M, N)$ and $\operatorname{Ext}_{R}^{i}(M, N)$.

Proof. Let $P \rightarrow M$ be a projective resolution, then $h$ lifts to an element of $D_{A}(R, P)$, and by Lemma $2.6 h$ lifts also to $D_{A}\left(R, \operatorname{Tor}_{i}^{R}(M, N)\right)$ and $D_{A}\left(R, \operatorname{Ext}_{R}^{i}(M, N)\right)$.

Joining Theorem 4.1 and Example 2.5 we get a new proof of the following classical result.

Corollary 4.3 (R. Hart [10]). Let $A$ be a commutative ring and $R$ a finitely generated commutative A-algebra. Then, every A-derivation of $R$ preserves the Fitting ideals of $\Omega_{R / A}$.

More generally, taking the Leibniz extension of the Lie derivative we also obtain that every $A$-derivation of $R$ preserves the Fitting ideals of $\wedge_{R}^{n} \Omega_{R / A}$ for every $n$. Notice also that the Leibniz extension of the Lie derivative commutes with the de Rham differential.

Example 4.4. Assume that $R$ is a principal ideal domain and that $M$ is a finitely generated $R$-module. Then, a derivation $h \in \operatorname{Der}_{A}(R, R)$ lifts to $D_{A}(R, M)$ if and only if $h$ preserves the Fitting ideals of $M$. In fact, we have a cyclic decomposition

$$
M=\frac{R}{\left(a_{1}\right)} e_{1} \oplus \cdots \oplus \frac{R}{\left(a_{n}\right)} e_{n}, \quad a_{i} \mid a_{i+1},
$$

and therefore the Fitting ideals are

$$
\left(a_{1} a_{2} \cdots a_{n}\right) \subset \cdots\left(a_{1} a_{2}\right) \subset\left(a_{1}\right) .
$$


Assume that $h$ preserves all the Fitting ideals, then $h\left(a_{i}\right) \in\left(a_{i}\right)$ for every $i$; this is clear if either $i=1$ or $a_{i}=0$, while for $i>1$ and $a_{i} \neq 0$ we have

$$
\begin{gathered}
h\left(a_{1} \cdots a_{i-1}\right) \in\left(a_{1} \cdots a_{i-1}\right), \quad h\left(a_{1} \cdots a_{i}\right) \in\left(a_{1} \cdots a_{i}\right), \\
h\left(a_{1} \cdots a_{i}\right)=h\left(a_{1} \cdots a_{i-1}\right) a_{i}+a_{1} \cdots a_{i-1} h\left(a_{i}\right),
\end{gathered}
$$

and then

$$
a_{1} \cdots a_{i-1} h\left(a_{i}\right) \in\left(a_{1} \cdots a_{i-1} a_{i}\right) .
$$

Now the map

$$
u: M \rightarrow M, \quad u\left(\sum x_{i} e_{i}\right)=\sum h\left(x_{i}\right) e_{i}
$$

gives a derivation of pair $(h, u)$.

\section{Coherent sheaves of DG-Lie algebras AND trace maps}

Assume now that $X \rightarrow \operatorname{Spec} A$ is a scheme over $A$, and denote by

$$
\Theta_{X / A}=\mathcal{H} m_{\mathcal{O}_{X}}\left(\Omega_{X / A}, \mathcal{O}_{X}\right)=\operatorname{Der}_{A}\left(\mathcal{O}_{X}, \mathcal{O}_{X}\right)
$$

the relative tangent sheaf. Given a sheaf $\mathcal{F}$ of $\mathcal{O}_{X}$-modules, we can define $\mathcal{D}_{A}(X, \mathcal{F})$ as the subsheaf of $\Theta_{X / A} \times \mathcal{H} m_{A}(\mathcal{F}, \mathcal{F})$ of all the elements satisfying the same condition of Definition 2.1. Similarly, for every morphism $f: \mathcal{F} \rightarrow \mathcal{G}$ of sheaves of $\mathcal{O}_{X}$-modules we can define the sheaf $\mathcal{D}_{A}(X, \mathcal{F} \stackrel{f}{\rightarrow} \mathcal{G})$, for instance by the exact sequence:

$0 \rightarrow \mathcal{D}_{A}(X, \mathcal{F} \stackrel{f}{\rightarrow} \mathcal{G}) \rightarrow \mathcal{D}_{A}(X, \mathcal{F}) \times_{\Theta_{X / A}} \mathcal{D}_{A}(X, \mathcal{G}) \stackrel{\Phi}{\rightarrow} \mathcal{H}_{0} m_{\mathcal{O}_{X}}(\mathcal{F}, \mathcal{G}), \quad \Phi(h, u, v)=f u-v f$.

If $\mathcal{F}$ is coherent and $p: Y \rightarrow X$ is the affine morphism of schemes such that $p_{*} \mathcal{O}_{Y}=$ $\mathcal{O}_{X} \oplus \mathcal{F}$, see e.g., [11, Exercise II.5.17], then by Lemma 2.3 we have an exact sequence of sheaves of $\mathcal{O}_{X}$-modules

$$
0 \rightarrow \mathcal{D}_{A}(X, \mathcal{F}) \rightarrow p_{*} \Theta_{Y / A} \rightarrow \mathcal{H} m_{\mathcal{O}_{X}}\left(\mathcal{F}, \mathcal{O}_{X}\right) \oplus \mathcal{H} m_{\mathcal{O}_{X}}\left(\Omega_{X / A}, \mathcal{F}\right) .
$$

Proposition 5.1. In the above situation, if $A$ is noetherian, $X$ of finite type over $A$ and $\mathcal{F}$ and $\mathcal{G}$ coherent sheaves, then also $\mathcal{D}_{A}(X, \mathcal{F}), \mathcal{D}_{A}(X, \mathcal{G})$ and $\mathcal{D}_{A}(X, \mathcal{F} \stackrel{f}{\rightarrow} \mathcal{G})$ are coherent. For every open affine subset $U \subset X$, we have:

$\mathcal{D}_{A}(X, \mathcal{F})(U) \cong D_{A}\left(\mathcal{O}_{X}(U), \mathcal{F}(U)\right), \quad \mathcal{D}_{A}(X, \mathcal{F} \stackrel{f}{\rightarrow} \mathcal{G})(U) \cong D_{A}\left(\mathcal{O}_{X}(U), \mathcal{F}(U) \stackrel{f}{\rightarrow} \mathcal{G}(U)\right)$.

Proof. According to the above assumptions, the scheme $Y$ is also of finite type over $A$ and the morphism $p$ is finite. In particular, both $\Omega_{X / A}$ and $p_{*} \Theta_{Y / A}$ are coherent sheaves and so $\mathcal{D}_{A}(X, \mathcal{F})$ is coherent, being the kernel of a morphism of coherent sheaves. Given an open affine subset $U \subset X$, the proof that the natural map $\mathcal{D}_{A}(X, \mathcal{F})(U) \rightarrow D_{A}\left(\mathcal{O}_{X}(U), \mathcal{F}(U)\right)$ is an isomorphism follows by comparing the exact sequences obtained by applying the functor $\Gamma(U,-)$ to $(5.1)$ and the exact sequence of Lemma 2.3. The statement about $\mathcal{D}_{A}(X, \mathcal{F} \stackrel{f}{\rightarrow} \mathcal{G})$ is proved in the same way.

Example 5.2. Let $\mathcal{F}$ be a locally free sheaf on $X$, then the sheaf $\mathcal{D}_{A}(X, \mathcal{F})$ is isomorphic to the sheaf of $A$-linear first order differential operators on $\mathcal{F}$ with scalar symbol. Via this isomorphism, the exact sequence

$$
0 \rightarrow \mathcal{H}^{\circ o m_{\mathcal{O}_{X}}}(\mathcal{F}, \mathcal{F}) \rightarrow \mathcal{D}_{A}(X, \mathcal{F}) \rightarrow \Theta_{X / A} \rightarrow 0
$$

corresponds, up to isomorphism, to the Atiyah extension of $\mathcal{F}$, cf. [2], [20, Example 2.3], [25, p. 145]. 
Definition 5.3. Let $X \rightarrow \operatorname{Spec} A$ be a noetherian scheme over a noetherian ring $A$. By a (quasi)coherent sheaf of Lie algebras over $X / A$ we mean a (quasi)coherent sheaf of $\mathcal{O}_{X^{-}}$ modules $\mathcal{L}$, together an $A$-bilinear bracket $\mathcal{L} \times \mathcal{L} \stackrel{[-,-]}{\longrightarrow} \mathcal{L}$ inducing a structure of Lie algebra over $A$ on every stalk of $\mathcal{L}$. Note that the bracket is not $\mathcal{O}_{X^{-}}$linear, so this is not the same structure as a Lie algebra in the monoidal category of quasi coherent module. A morphism of (quasi)coherent sheaves of Lie algebras is a morphism of sheaves of $\mathcal{O}_{X}$-modules commuting with the brackets.

For instance, if $\mathcal{F}$ is a coherent sheaf on $X$, then the anchor map $\alpha: \mathcal{D}_{A}(X, \mathcal{F}) \rightarrow \Theta_{X / A}$ is a morphism of coherent sheaves of Lie algebras over $X / A$. If $\mathcal{F} \rightarrow \mathcal{G}$ is a morphism of coherent sheaves, then the natural maps

$$
\mathcal{D}_{A}(X, \mathcal{F} \rightarrow \mathcal{G}) \rightarrow \mathcal{D}_{A}(X, \mathcal{F}), \quad \mathcal{D}_{A}(X, \mathcal{F} \rightarrow \mathcal{G}) \rightarrow \mathcal{D}_{A}(X, \mathcal{G}),
$$

are morphisms of coherent sheaves of Lie algebras over $X / A$.

Recall that a differential graded (DG) Lie algebra over a commutative ring $A$ is the data of a cochain complex $(L, d)$ of $A$-modules, together with an $A$-bilinear map $[-,-]: L \times L \rightarrow L$ (called bracket) of degree 0 , such that the following conditions are satisfied:

(1) (graded skewsymmetry) $[x, y]=-(-1)^{i j}[y, x] \in L^{i+j}$, for every $x \in L^{i}$ and $y \in L^{j}$;

(2) $[x, x]=0$ for every $x \in L^{2 i}$, and $[x,[x, x]]=0$ for every $x \in L^{2 i+1}$, with $i \in \mathbb{Z}$;

(3) (graded Jacobi identity) $[x,[y, z]]=[[x, y], z]+(-1)^{i j}[y,[x, z]]$, for every $x \in L^{i}$, $y \in L^{j}$ and $z \in L$

(4) (graded Leibniz rule) $d[x, y]=[d x, y]+(-1)^{i}[x, d y]$, for every $x \in L^{i}$ and $y \in L^{j}$.

A morphism of differential graded Lie algebras $\chi: L \rightarrow M$ is a morphism of cochain complexes that commutes with brackets.

Definition 5.4. Let $X \rightarrow \operatorname{Spec} A$ be a noetherian scheme over a noetherian ring $A$. By a quasi-coherent sheaf of DG-Lie algebras over $X / A$ we mean a complex $\mathcal{L}^{*}$ of quasi-coherent sheaves of $\mathcal{O}_{X}$-modules, together an $A$-bilinear bracket $\mathcal{L}^{*} \times \mathcal{L}^{*} \stackrel{[-,-]}{\longrightarrow} \mathcal{L}^{*}$ inducing a structure of DG-Lie algebra over $A$ on every stalk of $\mathcal{L}$. A quasi-coherent sheaf of DG-Lie algebras $\mathcal{L}^{*}$ is coherent if $\oplus \mathcal{L}^{i}$ is a coherent sheaf. A morphism of quasi-coherent sheaves of DG-Lie algebras is a morphism of complexes of $\mathcal{O}_{X}$-modules commuting with the brackets.

Example 5.5. Let $X \rightarrow \operatorname{Spec} A$ be a noetherian scheme over a noetherian $\operatorname{ring} A$, and let $\mathcal{E}^{*}$ be a bounded complex of coherent sheaves of $\mathcal{O}_{X}$-modules. For every index $i$, the sheaf

$$
\mathcal{H}^{\prime} m_{\mathcal{O}_{X}}^{i}\left(\mathcal{E}^{*}, \mathcal{E}^{*}\right)=\prod_{j} \mathcal{H} m_{\mathcal{O}_{X}}\left(\mathcal{E}^{j}, \mathcal{E}^{j+i}\right)
$$

is coherent and then $\mathcal{H}_{0} m_{\mathcal{O}_{X}}^{*}\left(\mathcal{E}^{*}, \mathcal{E}^{*}\right)$ is a coherent sheaf of DG-Lie algebras.

As in Section 3, we can define the complex of $\mathcal{O}_{X}$-modules $\mathcal{D}_{A}^{*}\left(X, \mathcal{E}^{*}\right)$ as the subsheaf of $\operatorname{Der}_{A}^{*}\left(\mathcal{O}_{X}, \mathcal{O}_{X}\right) \times \mathcal{H} o m_{A}^{*}\left(\mathcal{E}^{*}, \mathcal{E}^{*}\right)$, whose elements are the pairs $(h, u)$ such that

$$
u(r m)-r u(m)=h(r) m, \quad \text { for every } r \in \mathcal{O}_{X}, m \in \mathcal{E}^{*} .
$$

For every $i \neq 0$ we have $\mathcal{D}_{A}^{i}\left(X, \mathcal{E}^{*}\right)=\mathcal{H} m_{\mathcal{O}_{X}}^{i}\left(\mathcal{E}^{*}, \mathcal{E}^{*}\right)$, while $\mathcal{D}_{A}^{0}\left(X, \mathcal{E}^{*}\right)$ is the limit of the set of anchor maps $\mathcal{D}_{A}^{0}\left(X, \mathcal{E}^{j}\right) \stackrel{\alpha}{\rightarrow} \operatorname{Der}_{A}\left(\mathcal{O}_{X}, \mathcal{O}_{X}\right)=\Theta_{X / A}$. Thus $\mathcal{D}_{A}^{*}\left(X, \mathcal{E}^{*}\right)$ is a coherent sheaf of DG-Lie algebras and there exists a short exact sequence of coherent sheaves of DG-Lie algebras over $X / A$ :

$$
0 \rightarrow \mathcal{H o m}_{\mathcal{O}_{X}}^{*}\left(\mathcal{E}^{*}, \mathcal{E}^{*}\right) \rightarrow \mathcal{D}_{A}^{*}\left(X, \mathcal{E}^{*}\right) \stackrel{\alpha}{\longrightarrow} \Theta_{X / A} .
$$

According to Lemma 3.1 , the anchor map $\mathcal{D}_{A}^{*}\left(X, \mathcal{E}^{*}\right) \stackrel{\alpha}{\longrightarrow} \Theta_{X / A}$ is surjective whenever every sheaf $\mathcal{E}^{j}$ is locally free. Analogously to Remark 5.1, for every open affine subset $U \subset X$, we have:

$$
\mathcal{D}_{A}\left(X, \mathcal{E}^{*}\right)(U) \cong D_{A}\left(\mathcal{O}_{X}(U), \mathcal{E}^{*}(U)\right) \text {. }
$$


It is well known that for every bounded complex $\mathcal{E}^{*}$ of locally free sheaves we can define the trace map

$$
\operatorname{Tr}: \mathcal{H}_{0}{ }_{\mathcal{O}_{X}}^{*}\left(\mathcal{E}^{*}, \mathcal{E}^{*}\right) \rightarrow \mathcal{O}_{X}
$$

which is a surjective morphism of complexes of coherent sheaves: given $f \in \mathcal{H}_{0} m_{\mathcal{O}_{X}}^{i}\left(\mathcal{E}^{*}, \mathcal{E}^{*}\right)$ then $\operatorname{Tr}(f)=0$ for $i \neq 0$; if $i=0$, then $f$ is the datum of a finite sequence of morphisms of locally free sheaves $f_{j}: \mathcal{E}^{j} \rightarrow \mathcal{E}^{j}$, and

$$
\operatorname{Tr}(f)=\sum_{j}(-1)^{j} \operatorname{Tr}\left(f_{j}\right)
$$

where $\operatorname{Tr}: \mathcal{H} m_{\mathcal{O}_{X}}\left(\mathcal{E}^{j}, \mathcal{E}^{j}\right) \rightarrow \mathcal{O}_{X}$ is the usual trace map.

It is easy to verify that $\mathrm{Tr}$ is also a morphism of sheaves of DG-Lie algebras, where $\mathcal{O}_{X}$ is equipped with the trivial bracket. In fact, since the differential on $\mathcal{H}_{0} m_{\mathcal{O}_{X}}^{*}\left(\mathcal{E}^{*}, \mathcal{E}^{*}\right)$ is an adjoint operator, it is sufficient to prove that $\operatorname{Tr}([f, g])=0$ for every $f \in \mathcal{H}^{\prime} m_{\mathcal{O}_{X}}^{i}\left(\mathcal{E}^{*}, \mathcal{E}^{*}\right)$ and $g \in \mathcal{H o m}_{\mathcal{O}_{X}}^{j}\left(\mathcal{E}^{*}, \mathcal{E}^{*}\right)$. This is clear if $i+j \neq 0$, and so it is not restrictive to assume $i=-j$. Since the trace is $\mathcal{O}_{X}$-linear, we can reduce this fact to the following statement of linear algebra: let $V_{i}, i \in \mathbb{Z}$, be a sequence of finite dimensional vector spaces, with $V_{i} \neq 0$ for at most finitely many indices $i$. Then for every integer $p$ and any two sequences

$$
f_{i}: V_{i} \rightarrow V_{i+p}, \quad g_{i}: V_{i} \rightarrow V_{i-p},
$$

of linear maps, we have

$$
\sum_{i}(-1)^{i} \operatorname{Tr}\left(f_{i-p} g_{i}-(-1)^{p^{2}} g_{i+p} f_{i}\right)=0 .
$$

In fact, by a basic fact of linear algebra we have $\operatorname{Tr}\left(f_{i-p} g_{i}\right)=\operatorname{Tr}\left(g_{i} f_{i-p}\right)$ for every $i$, and then

$$
\begin{aligned}
\sum_{i}(-1)^{i} \operatorname{Tr}\left(f_{i-p} g_{i}-(-1)^{p^{2}} g_{i+p} f_{i}\right) & =\sum_{i}(-1)^{i} \operatorname{Tr}\left(f_{i-p} g_{i}\right)-(-1)^{i+p} \operatorname{Tr}\left(g_{i+p} f_{i}\right) \\
& =\sum_{i}(-1)^{i} \operatorname{Tr}\left(f_{i-p} g_{i}\right)-(-1)^{i} \operatorname{Tr}\left(g_{i} f_{i-p}\right)=0 .
\end{aligned}
$$

Denoting by $\mathcal{H}_{0} m_{\mathcal{O}_{X}}^{*}\left(\mathcal{E}^{*}, \mathcal{E}^{*}\right)_{0}$ the sheaf of traceless endomorphisms of $\mathcal{E}^{*}$, i.e., the kernel of (5.4), and by $\operatorname{Ext}_{X}^{i}(\mathcal{F}, \mathcal{F})_{0}$ its hypercohomology groups, there exists a long exact sequence

$$
\rightarrow \operatorname{Ext}_{X}^{1}(\mathcal{F}, \mathcal{F}) \stackrel{\operatorname{Tr}}{\longrightarrow} H^{1}\left(X, \mathcal{O}_{X}\right) \rightarrow \operatorname{Ext}_{X}^{2}(\mathcal{F}, \mathcal{F})_{0} \rightarrow \operatorname{Ext}_{X}^{2}(\mathcal{F}, \mathcal{F}) \stackrel{\operatorname{Tr}}{\longrightarrow} H^{2}\left(X, \mathcal{O}_{X}\right) \rightarrow
$$

By the results of Section 2, we can extend the trace map Tr: $\mathcal{H}_{0} m_{\mathcal{O}_{X}}^{0}\left(\mathcal{E}^{*}, \mathcal{E}^{*}\right) \rightarrow \mathcal{O}_{X}$ to a Lie algebra morphism

$$
\operatorname{Tr}: \mathcal{D}_{A}^{0}\left(X, \mathcal{E}^{*}\right) \rightarrow \mathcal{D}_{A}\left(X, \operatorname{det} \mathcal{E}^{*}\right) .
$$

Assume that the finite complex $\mathcal{E}^{*}$ is nonzero only for degree between $n$ and $m$, then by definition, an element of $\mathcal{D}_{A}^{0}\left(X, \mathcal{E}^{*}\right)$ is given by a sequence $\left(h, u_{n}, \ldots, u_{m}\right)$ where $h \in$ $\operatorname{Der}_{A}\left(\mathcal{O}_{X}, \mathcal{O}_{X}\right)$ and $\left(h, u_{i}\right) \in \mathcal{D}_{A}\left(X, \mathcal{E}^{i}\right)$ for every $i$. Taking the trace of every pair $\left(h, u_{i}\right)$ (Definition 2.8) we obtain a finite sequence of derivations of pairs

$$
\left(h, v_{i}\right) \in \mathcal{D}_{A}\left(X, \operatorname{det} \mathcal{E}^{i}\right), \quad v_{i}=\operatorname{Tr}\left(u_{i}\right), \quad i=n, \ldots, m .
$$

Then, for every odd index $i$ we consider the transpose (2.4)

$$
\left(h, v_{i}\right)^{T} \in \mathcal{D}_{A}\left(X,\left(\operatorname{det} \mathcal{E}^{i}\right)^{-1}\right) .
$$

Since

$$
\left[\left(h, v_{0}\right),\left(h, v_{1}\right)^{T}\right] \in \mathcal{D}_{A}\left(X, \operatorname{det} \mathcal{E}^{0}\right) \times_{\operatorname{Der}_{A}\left(\mathcal{O}_{X}, \mathcal{O}_{X}\right)} \mathcal{D}_{A}\left(X,\left(\operatorname{det} \mathcal{E}^{1}\right)^{-1}\right)
$$


we may apply the Lie morphism $\Phi$ of Lemma 2.6 in order to obtain an element $\operatorname{Tr}\left(h, u_{0}, u_{1}\right) \in$ $\mathcal{D}_{A}\left(X,\left(\operatorname{det} \mathcal{E}^{0}\right) \otimes\left(\operatorname{det} \mathcal{E}^{1}\right)^{-1}\right)$. It is now clear that, with a finite number of constructions as above, we have a well defined element

$$
\operatorname{Tr}\left(h, u_{n}, \ldots, u_{m}\right) \in \mathcal{D}_{A}\left(X, \operatorname{det} \mathcal{E}^{*}\right)=\mathcal{D}_{A}\left(X, \bigotimes_{i=n}^{m}\left(\operatorname{det} \mathcal{E}^{i}\right)^{(-1)^{i}}\right) .
$$

Moreover, we have also proved that all the above considered maps

$$
\mathcal{D}_{A}\left(X, \varepsilon^{i}\right) \rightarrow \mathcal{D}_{A}\left(X, \operatorname{det} \varepsilon^{i}\right), \quad \prod_{i}^{\times} \mathcal{D}_{A}\left(X, \operatorname{det} \mathcal{E}^{i}\right) \rightarrow \mathcal{D}_{A}\left(X, \operatorname{det} \mathcal{E}^{*}\right),
$$

are morphisms of sheaves of Lie algebras. Keeping in mind that

$$
\mathcal{D}_{A}^{0}\left(X, \mathcal{E}^{*}\right)=\prod_{i}^{\times} \mathcal{D}_{A}\left(X, \mathcal{E}^{i}\right), \quad \mathcal{D}_{A}^{j}\left(X, \mathcal{E}^{*}\right)=\mathcal{H}_{0} m_{\mathcal{O}_{X}}^{j}\left(\mathcal{E}^{*}, \mathcal{E}^{*}\right), \quad j \neq 0,
$$

we can glue together (5.6) and (5.4) and obtain a morphism of sheaves of DG-Lie algebras

$$
\operatorname{Tr}: \mathcal{D}_{A}^{*}\left(X, \mathcal{E}^{*}\right) \rightarrow \mathcal{D}_{A}\left(X, \operatorname{det} \mathcal{E}^{*}\right) .
$$

The following theorem is now clear.

Theorem 5.6. For every bounded complex of locally free sheaves $\mathcal{E}^{*}$ on a scheme $X$ of finite type over a noetherian ring A, there exists a commutative diagram of morphisms of coherent sheaves of $D G$-Lie algebras

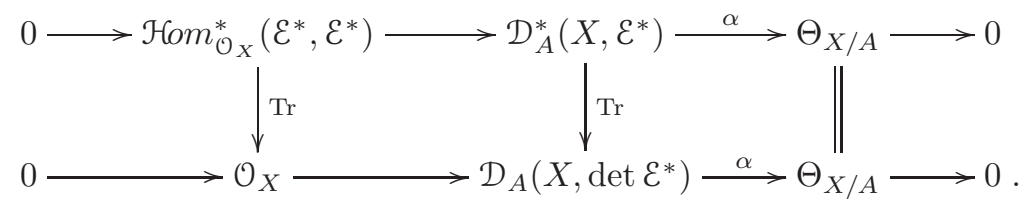

\section{A short Review of Deformation theory Via DG-Lie algebras}

The main references for this section are $[6,8,17,18,19]$. From this section, and throughout the rest of the paper, we work over a fixed algebraically closed field $\mathbb{K}$ of characteristic zero. We denote by Set the category of sets and by $\mathbf{A r t}_{\mathbb{K}}$ the category of Artin local $\mathbb{K}$ algebras with residue field $\mathbb{K}$, and by DGLA $=$ DGLA $\mathbf{A}_{\mathbb{K}}$ the category of DG-Lie algebras over $\mathbb{K}$. Unless otherwise specified, for every local algebra $A \in \mathbf{A r t}_{\mathbb{K}}$, we denote by $\mathfrak{m}_{A}$ its maximal ideal.

Given a DG-Lie algebra $L$ over $\mathbb{K}$, we can define two functors of Artin rings. The MaurerCartan functor $\mathrm{MC}_{L}: \mathbf{A r t}_{\mathbb{K}} \rightarrow$ Set is defined by:

$$
\operatorname{MC}_{L}(A)=\left\{x \in L^{1} \otimes \mathfrak{m}_{A} \mid d x+\frac{1}{2}[x, x]=0\right\}
$$

where the DG-Lie structure on $L \otimes \mathfrak{m}_{A}$ is obtained by scalar extension from the DG-Lie structure on $L$. The deformation functor $\operatorname{Def}_{L}: \mathbf{A r t}_{\mathbb{K}} \longrightarrow$ Set is:

$$
\operatorname{Def}_{L}(A)=\frac{\operatorname{MC}_{L}(A)}{\text { gauge }}=\frac{\left\{x \in L^{1} \otimes \mathfrak{m}_{A} \mid d x+\frac{1}{2}[x, x]=0\right\}}{\exp \left(L^{0} \otimes \mathfrak{m}_{A}\right)},
$$

where the gauge action $*: \exp \left(L^{0} \otimes \mathfrak{m}_{A}\right) \times \mathrm{MC}_{L}(A) \longrightarrow \mathrm{MC}_{L}(A)$ may be defined by the explicit formula

$$
e^{a} * x:=x+\sum_{n \geq 0} \frac{[a,-]^{n}}{(n+1) !}([a, x]-d a) .
$$


A quasi-isomorphism of DG-Lie algebras is a morphism that induces an isomorphism in cohomology. Two DG-Lie algebras $L$ and $M$ are said to be quasi-isomorphic, or homotopy equivalent, if they are equivalent under the equivalence relation generated by quasiisomorphisms.

By standard facts about deformation functors $[18,19]$, it is known that the tangent space to $\operatorname{Def}_{L}$ is isomorphic to $H^{1}(L)$ and that $H^{2}(L)$ is an obstruction space.

Remark 6.1. Every morphism $L \rightarrow M$ of DG-Lie algebras induces a natural transformation of the associated deformation functors $\operatorname{Def}_{L} \rightarrow \operatorname{Def}_{M}$. A basic result [19, Thoerema 1.5] asserts that if $H^{0}(L) \rightarrow H^{0}(M)$ is surjective, $H^{1}(L) \rightarrow H^{1}(M)$ is bijective and $H^{2}(L) \rightarrow$ $H^{2}(M)$ is injective, then $\operatorname{Def}_{L} \rightarrow \operatorname{Def}_{M}$ is an isomorphism.

Lemma 6.2. Let $L$ be a DG-Lie algebra over a field of characteristic 0 with $H^{1}(L)$ finite dimensional. If the natural map

$$
N^{0}=\left\{x \in L^{0} \mid d x=0,\left[x, L^{1}\right]=0\right\} \rightarrow H^{0}(L)
$$

is surjective, then $\operatorname{Def}_{L}$ is pro-representable.

Proof. Notice first that $N^{0}$ is a Lie subalgebra of $L^{0}$. Then define $N^{i}=L^{i}$ for every $i \geq 2$ and choose a vector subspace $N^{1} \subset L^{1}$ such that $L^{1}=d\left(L^{0}\right) \oplus N^{1}$. The inclusion of DG-Lie algebras $N=\oplus_{i \geq 0} N^{i} \hookrightarrow L$ satisfies the condition of Remark 6.1 and then $\operatorname{Def}_{L}=\operatorname{Def}_{N}$. On the other side the gauge action on $\mathrm{MC}_{N}$ is trivial and then $\operatorname{Def}_{N}=\mathrm{MC}_{N}$ is prorepresentable by Schlessinger's theorem [23, Thm. 2.11].

Let $\boldsymbol{\Delta}_{\text {mon }}$ be the category whose objects are finite ordinal sets and whose morphisms are order-preserving injective maps between them. A semicosimplicial differential graded Lie algebra is a covariant functor $\boldsymbol{\Delta}_{\text {mon }} \rightarrow$ DGLA. Equivalently, a semicosimplicial DG-Lie algebra $\mathfrak{g}^{\Delta}$ is a diagram

$$
\mathfrak{g}_{0} \Longrightarrow \mathfrak{g}_{1} \Longrightarrow \mathfrak{g}_{2} \equiv
$$

where each $\mathfrak{g}_{i}$ is a DG-Lie algebra, and for each $i>0$, there are $i+1$ morphisms of DG-Lie algebras

$$
\partial_{k, i}: \mathfrak{g}_{i-1} \rightarrow \mathfrak{g}_{i}, \quad k=0, \ldots, i,
$$

such that $\partial_{k+1, i+1} \partial_{l, i}=\partial_{l, i+1} \partial_{k, i}$, for any $k \geq l$. Here we use the non-standard notation of lower indexes for a semicosimplicial object, since the upper indexes are already used to denote degrees.

We denote by $\operatorname{Tot}\left(\mathfrak{g}^{\Delta}\right)$ the image of a semicosimplicial differential graded Lie algebra $\mathfrak{g}^{\Delta}$ via the Thom-Whitney totalization functor

$$
\text { Tot: DGLA }{ }^{\Delta_{\text {mon }}} \rightarrow \text { DGLA. }
$$

We refer to [3, Section2], [6, Section 3.2], [7, Section 3] and [15, Section 3] for an explicit description of the DG-Lie algebra $\operatorname{Tot}\left(\mathfrak{g}^{\Delta}\right)$ and its properties. Here, we remind only that there exists a quasi-isomorphism of complexes of vector spaces between $\operatorname{Tot}\left(\mathfrak{g}^{\Delta}\right)$ and the total complex associated with the cochain complex $C\left(\mathfrak{g}^{\Delta}\right)$ (considering any $\mathfrak{g}^{\Delta}$ as a semicosimplicial object in the abelian category of DG-vector spaces). As a consequence of this fact, if $f: \mathfrak{g}^{\Delta} \rightarrow \mathfrak{h}^{\Delta}$ is a morphism of semicosimplicial differential graded Lie algebras such that $f: \mathfrak{g}_{i} \rightarrow \mathfrak{h}_{i}$ is a quasi-isomorphism of DG-Lie algebras for every $i$, then the image $\operatorname{Tot}(f): \operatorname{Tot}\left(\mathfrak{g}^{\Delta}\right) \rightarrow \operatorname{Tot}\left(\mathfrak{h}^{\Delta}\right)$ is a quasi-isomorphism.

Considering the DG-Lie algebra $\operatorname{Tot}\left(\mathfrak{g}^{\Delta}\right)$, we can naturally associate with any semicosimplicial DG-Lie algebra $\mathfrak{g}^{\Delta}$ the functor of Artin rings $\operatorname{Def}_{\operatorname{Tot}_{\left(\mathfrak{g}^{\Delta}\right)}: \operatorname{Art}_{\mathbb{K}} \rightarrow \text { Set. According }}$ to [6, Definition 3.1 and Definition 3.3], we can also associate with $\mathfrak{g}^{\Delta}$ two other functors of Artin rings. The former

$$
Z_{\mathrm{sc}}^{1}\left(\exp \mathfrak{g}^{\Delta}\right): \mathbf{A r t}_{\mathbb{K}} \rightarrow \mathbf{S e t}
$$


is defined, for any $A \in \mathbf{A r t}_{\mathbb{K}}$, by

$$
Z_{\mathrm{sc}}^{1}\left(\exp \mathfrak{g}^{\Delta}\right)(A)=\left\{\begin{array}{l|l}
(l, m) \in\left(\mathfrak{g}_{0}^{1} \times \mathfrak{g}_{1}^{0}\right) \otimes \mathfrak{m}_{A} & \begin{array}{l}
d l+\frac{1}{2}[l, l]=0 \\
\partial_{1,1} l=e^{m} * \partial_{0,1} l, \\
\partial_{0,2} m \bullet-\partial_{1,2} m \bullet \partial_{2,2} m=d n+\left[\partial_{2,2} \partial_{0,1} l, n\right] \\
\text { for some } n \in \mathfrak{g}_{2}^{-1} \otimes \mathfrak{m}_{A}
\end{array}
\end{array}\right\} .
$$

The latter

$$
H_{\mathrm{sc}}^{1}\left(\exp \mathfrak{g}^{\Delta}\right): \mathbf{A r t}_{\mathbb{K}} \rightarrow \mathbf{S e t}
$$

is defined, for any $A \in \mathbf{A r t}_{\mathbb{K}}$, by

$$
H_{\mathrm{sc}}^{1}\left(\exp \mathfrak{g}^{\Delta}\right)(A)=Z_{\mathrm{sc}}^{1}\left(\exp \mathfrak{g}^{\Delta}\right)(A) / \sim,
$$

where two elements $\left(l_{0}, m_{0}\right)$ and $\left(l_{1}, m_{1}\right) \in Z_{\mathrm{sc}}^{1}\left(\exp ^{\Delta}\right)(A)$ are equivalent under the relation $\sim$ if and only if there exist elements $a \in \mathfrak{g}_{0}^{0} \otimes \mathfrak{m}_{A}$ and $b \in \mathfrak{g}_{1}^{-1} \otimes \mathfrak{m}_{A}$ such that

$$
\left\{\begin{array}{l}
e^{a} * l_{0}=l_{1} \\
-m_{0} \bullet-\partial_{1,1} a \bullet m_{1} \bullet \partial_{0,1} a=d b+\left[\partial_{0,1} l_{0}, b\right] .
\end{array}\right.
$$

Theorem 6.3. Let $\mathfrak{g}^{\Delta}$ be a semicosimplicial DG-Lie algebra such that $H^{j}\left(\mathfrak{g}_{i}\right)=0$ for all $i \geq 0$ and $j<0$. Then, there exists an isomorphism of functors of Artin rings

$$
\operatorname{Def}_{\operatorname{Tot}\left(\mathfrak{g}^{\Delta}\right)} \rightarrow H_{\mathrm{sc}}^{1}\left(\exp \mathfrak{g}^{\Delta}\right) .
$$

Proof. See [6, Theorem 4.10].

Remark 6.4. If each $\mathfrak{g}_{i}$ is concentrated in degree zero, i.e., $\mathfrak{g}^{\Delta}$ is a semicosimplicial Lie algebra, then the functor $H_{\mathrm{sc}}^{1}\left(\exp \mathfrak{g}^{\Delta}\right)$ has an easier explicit description [7, Section 1]:

$$
\begin{gathered}
H_{\mathrm{sc}}^{1}\left(\exp \mathfrak{g}^{\Delta}\right): \mathbf{A r t}_{\mathbb{K}} \rightarrow \text { Set } \\
H_{s c}^{1}\left(\exp \mathfrak{g}^{\Delta}\right)(A)=\frac{\left\{x \in \mathfrak{g}_{1} \otimes \mathfrak{m}_{A} \mid e^{\partial_{0} x} e^{-\partial_{1} x} e^{\partial_{2} x}=1\right\}}{\sim},
\end{gathered}
$$

where $x \sim y$ if and only if there exists $a \in \mathfrak{g}_{0} \otimes \mathfrak{m}_{A}$, such that $e^{-\partial_{1} a} e^{x} e^{\partial_{0} a}=e^{y}$.

Let $\mathcal{L}$ be a coherent sheaf of DG-Lie algebras over an algebraic variety $X$ and $\mathcal{U}=\left\{U_{i}\right\}$ an affine open cover of $X$. In this case, the Čech (double) complex $C(\mathcal{U}, \mathcal{L})$ of $\mathcal{L}$ is exactly the total cochain complex associated with the semicosimplicial DG-Lie algebra:

$$
\mathcal{L}(\mathcal{U}): \quad \prod_{i} \mathcal{L}\left(U_{i}\right) \Longrightarrow \prod_{i, j} \mathcal{L}\left(U_{i j}\right) \Longrightarrow \prod_{i, j, k} \mathcal{L}\left(U_{i j k}\right) \rightrightarrows \equiv,
$$

where the face operators $\partial_{h}: \prod_{i_{0}, \ldots, i_{k-1}} \mathcal{L}\left(U_{i_{0} \cdots i_{k-1}}\right) \rightarrow \prod_{i_{0}, \ldots, i_{k}} \mathcal{L}\left(U_{i_{0} \cdots i_{k}}\right)$ are given by

$$
\partial_{h}(x)_{i_{0} \ldots i_{k}}=x_{i_{0} \ldots \widehat{i_{h} \ldots i_{k} \mid U_{i_{0} \cdots i_{k}}}}, \quad \text { for } h=0, \ldots, k .
$$

For notational convenience, we denote by $\operatorname{Tot}(\mathcal{U}, \mathcal{L})$ the Thom-Whitney totalization of the semicosimplicial DG-Lie algebra $\mathcal{L}(\mathcal{U})$. Note that in this case

$$
H^{i}(\operatorname{Tot}(\mathcal{U}, \mathcal{L}))=H^{i}(C(\mathcal{U}, \mathcal{L}))=\check{\mathbb{H}}^{i}(\mathcal{U}, \mathcal{L})=\mathbb{H}^{i}(X, \mathcal{L}) .
$$

Remark 6.5. For any coherent sheaf of DG-Lie algebras $\mathcal{L}$, the quasi-isomorphism class of $\operatorname{Tot}(\mathcal{U}, \mathcal{L})$ does not depend on the choice of the affine open cover [6]. If $\mathcal{L} \rightarrow \mathcal{G}$ is a morphism of coherent sheaves of DG-Lie algebras that is a quasi-isomorphism at every stalk, then for any affine open cover $\mathcal{U}$, the induced morphism of DG-Lie algebras $\operatorname{Tot}(\mathcal{U}, \mathcal{L}) \rightarrow \operatorname{Tot}(\mathcal{U}, \mathcal{G})$ is a quasi-isomorphism of DG-Lie algebras.

This motivates the following definition. 
Definition 6.6. Let $\mathcal{L}$ be a (quasi) coherent sheaf of DG-Lie algebras over an algebraic variety $X$. Suppose that, for an open affine cover $\mathcal{U}$ of $X$ (and so for all), the DG-Lie algebra $\operatorname{Tot}(\mathcal{U}, \mathcal{L})$ controls a given deformation problem, then we also say that $\mathcal{L}$ controls the deformation problem. Note that in this case $\mathbb{H}^{1}(X, \mathcal{L})$ is the tangent space and $\mathbb{H}^{2}(X, \mathcal{L})$ is an obstructions space for the controlled deformation problem.

\section{Deformations of pairs (scheme, Coherent sheaf)}

From now on, unless otherwise specified, we denote by $X$ a scheme of finite type over the field $\mathbb{K}$.

Definition 7.1. Let $\mathcal{F}$ be a coherent sheaf on $X$. An infinitesimal deformation of the pair $(X, \mathcal{F})$ over $A \in \mathbf{A r t}_{\mathbb{K}}$ is the data $\left(X_{A}, \mathcal{F}_{A}\right)$ where:

- $X_{A}$ is an infinitesimal deformation of $X$ over $A$, i.e., a pull-back diagram

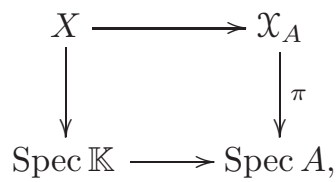

where $\pi$ is flat;

- $\mathcal{F}_{A}$ is a coherent sheaf of $\mathcal{O}_{X_{A}}$-modules on $X_{A}$, flat over $A$ and a morphism $\mathcal{F}_{A} \rightarrow \mathcal{F}$, inducing an isomorphism $\mathcal{F}_{A} \otimes_{\mathcal{O}_{X_{A}}} \mathcal{O}_{X} \rightarrow \mathcal{F}$.

Definition 7.2. Let $\mathcal{F}$ be a coherent sheaf on a scheme $X$. Two infinitesimal deformations $\left(X_{A}, \mathcal{F}_{A}\right)$ and $\left(X_{A}^{\prime}, \mathcal{F}_{A}^{\prime}\right)$ of the pair $(X, \mathcal{F})$ over $A$ are isomorphic, if there exist an isomorphism of deformations $f: X_{A} \rightarrow X_{A}^{\prime}$ and an isomorphism $\mathcal{F}_{A} \rightarrow f^{*} \mathcal{F}_{A}^{\prime}$ of coherent sheaves of $\mathcal{O}_{x_{A}}$-modules.

We recall that the trivial infinitesimal deformation of $(X, \mathcal{F})$ over $A \in \mathbf{A r t}_{\mathbb{K}}$ is given by the pair $\left(X \times \operatorname{Spec} A, \mathcal{F} \otimes_{\mathcal{O}} \mathcal{O}_{x \times \operatorname{Spec} \mathcal{A}}=\mathcal{F} \otimes_{\mathbb{K}} A\right)$.

An infinitesimal deformation of $(X, \mathcal{F})$ is locally trivial, if it is locally, in $X$, isomorphic to the trivial infinitesimal deformation.

Definition 7.3. Let $\mathcal{F}$ be a coherent sheaf on a scheme $X$. The functor of infinitesimal deformations of the pair $(X, \mathcal{F})$ is the functor

$$
\begin{gathered}
\operatorname{Def}_{(X, \mathcal{F})}: \operatorname{Art}_{\mathbb{K}} \rightarrow \text { Set, } \\
\operatorname{Def}_{(X, \mathcal{F})}(A)=\{\text { isomorphism classes of deformations of the pair }(X, \mathcal{F}) \text { over } A\} .
\end{gathered}
$$

The functor of locally trivial infinitesimal deformations of the pair $(X, \mathcal{F})$ is the functor

$$
\operatorname{Def}_{(X, F)}^{l t}: \operatorname{Art}_{\mathbb{K}} \rightarrow \text { Set }
$$

$\operatorname{Def}_{(X, F)}^{\mathrm{lt}}(A)=\{$ isomorphism classes of locally trivial deformations of the pair $(X, \mathcal{F})$ over $A\}$.

Remark 7.4. If $X$ is a smooth variety, then any infinitesimal deformation of $X$ is locally trivial. If $\mathcal{F}$ is a locally free sheaf over a variety $X$ then any infinitesimal deformation of the sheaf is locally trivial. Then, there exists an isomorphism of functors $\operatorname{Def}_{(X, \mathcal{F})}^{{ }^{\mathrm{lt}}} \cong \operatorname{Def}_{(X, \mathcal{F})}$, for a locally free sheaf $\mathcal{F}$ on a smooth variety $X$. 
7.1. Locally trivial infinitesimal deformations. Let us analyse the data that define a locally trivial infinitesimal deformation $\left(X_{A}, \mathcal{F}_{A}\right)$ of $(X, \mathcal{F})$ over $A$. Let $\mathcal{U}=\left\{U_{i}\right\}_{i \in I}$ be an affine open cover of $X$. The deformation $X_{A}$ of $X$ is trivial over every affine open subset; thus for any $i \in I$ there exists an isomorphism

$$
\alpha_{i}: \mathcal{O}_{X}\left(U_{i}\right) \otimes A \rightarrow \mathcal{O}_{X_{A}}\left(U_{i}\right) .
$$

On every $U_{i} \times \operatorname{Spec} A$, we have the sheaf $\mathcal{F}_{\mid U_{i}} \otimes A$ of $\mathcal{O}_{X}\left(U_{i}\right) \otimes A$-modules. Since $\alpha_{i}$ is an isomorphism, we can view $\mathcal{F}_{\mid U_{i}} \otimes A$ as a sheaf of $\mathcal{O}_{X_{A}}\left(U_{i}\right)$-modules; the $\mathcal{O}_{X_{A}}\left(U_{i}\right)$-modules structure on $\mathcal{F}_{\mid U_{i}} \otimes A$ is given by: $s \cdot x=\alpha_{i}{ }^{-1}(s) x$, for any $s \in \mathcal{O}_{x_{A}}\left(U_{i}\right)$ and $x \in \mathcal{F}_{\mid U_{i}} \otimes A$.

To give a locally trivial deformation $\mathcal{F}_{A}$ of the sheaf $\mathcal{F}$ over $X_{A}$, we need to glue the sheaves $\mathcal{F}_{\mid U_{i}} \otimes A$ of $\mathcal{O}_{X_{A}}\left(U_{i}\right)$-modules over double intersections. The isomorphisms

$$
\alpha_{i \mid U_{i j}}: \mathcal{O}_{X}\left(U_{i j}\right) \otimes A \rightarrow \mathcal{O}_{X_{A}}\left(U_{i j}\right) \quad \text { and } \quad \alpha_{j \mid U_{i j}}: \mathcal{O}_{X}\left(U_{i j}\right) \otimes A \rightarrow \mathcal{O}_{X_{A}}\left(U_{i j}\right)
$$

induce a structure of sheaf of $\mathcal{O}_{X_{A}}\left(U_{i j}\right)$-module on the sheaves $\mathcal{F}_{\mid U_{i}} \otimes A_{\mid U_{i j}}$ and $\mathcal{F}_{\mid U_{i}} \otimes A_{\mid U_{i j}}$, respectively. To glue these sheaves, we need an isomorphism $\psi_{i j}: \mathcal{F}_{\mid U_{i}} \otimes A_{\mid U_{i j}} \rightarrow \mathcal{F}_{\mid U_{i}} \otimes A_{\mid U_{i j}}$ of $\mathcal{O}_{X_{A}}\left(U_{i j}\right)$-modules, i.e., for all $s \in \mathcal{O}_{X_{A}}\left(U_{i j}\right), \psi_{i j}(s \cdot x)=s \cdot \psi(x)$. By the explicit definition of $\mathcal{O}_{X_{A}}\left(U_{i j}\right)$-modules structures, we have

$$
\psi_{i j}(s \cdot x)=\psi_{i j}\left(\alpha_{i \mid U_{i j}}^{-1}(s) x\right) \quad \text { and } \quad s \cdot \psi_{i j}(x)=\alpha_{j \mid U_{i j}}^{-1}(s) \psi_{i j}(x) .
$$

Since $\alpha_{i \mid U_{i j}}$ is an isomorphism, there exists $t \in \mathcal{O}_{X}\left(U_{i j}\right) \otimes A$ such that $s=\alpha_{i \mid U_{i j}}(t)$. Therefore, the linearity reads as follows

$$
\psi_{i j}(t x)=\alpha_{j \mid U_{i j}}^{-1} \alpha_{i \mid U_{i j}}(t) \psi_{i j}(x),
$$

for any $t \in \mathcal{O}_{X}\left(U_{i j}\right) \otimes A$ and $x \in \mathcal{F}_{\mid U_{i j}} \otimes A$.

In conclusion, in order to define a locally trivial infinitesimal deformation of $(X, \mathcal{F})$ over $A$, it is enough to give, for any $U_{i j}$, an automorphism $\theta_{i j}=\alpha_{j \mid U_{i j}}^{-1} \alpha_{i \mid U_{i j}}$ of $\mathcal{O}_{X}\left(U_{i j}\right) \otimes$ $A$, satisfying the cocycle condition on the triple intersections, an automorphism $\psi_{i j} \in$ $\operatorname{Hom}_{\mathbb{K}}\left(\mathcal{F}_{\mid U_{i j}} \otimes A, \mathcal{F}_{\mid U_{i j}} \otimes A\right)$ satisfying the cocycle condition on the triple intersections and such that $\psi_{i j}(t x)=\theta_{i j}(t)(x)$, for any $t \in \mathcal{O}_{X}\left(U_{i j}\right) \otimes A$ and $x \in \mathcal{F}_{\mid U_{i j}} \otimes A$.

Since we are in characteristic zero, according to Lemma 2.10, we can take the logarithm and conclude that $\left(\theta_{i j}, \psi_{i j}\right)=e^{x_{i j}}$, where $x_{i j} \in \mathcal{D}_{\mathbb{K}}(X, \mathcal{F})\left(U_{i j}\right) \otimes \mathfrak{m}_{A}$; the condition of gluing on triple intersections is equivalent to

$$
e^{x_{j k}} e^{-x_{i k}} e^{x_{i j}}=1 \in \exp \left(\mathcal{D}_{\mathbb{K}}(X, \mathcal{F})\left(U_{i j k}\right) \otimes \mathfrak{m}_{A}\right), \quad \forall i, j, k \in I .
$$

As regard the isomorphisms, suppose that $\left(X_{A}, \mathcal{F}_{A}\right)$ and $\left(X_{A}^{\prime}, \mathcal{F}_{A}^{\prime}\right)$ are locally trivial isomorphic infinitesimal deformations of the pair $(X, \mathcal{F})$ over $A$. Then, there exist an isomorphism of deformations $f: X_{A} \rightarrow X_{A}^{\prime}$ and an isomorphism $\phi: \mathcal{F}_{A} \rightarrow f^{*} \mathcal{F}_{A}^{\prime}$ of coherent sheaves of $\mathcal{O}_{X_{A}}$-modules.

Let $\mathcal{U}=\left\{U_{i}\right\}_{i \in I}$ be an affine open cover of $X$. Then, for each $i \in I$, there exists an induced automorphism of $\mathcal{O}_{X}\left(U_{i}\right) \otimes A$ :

$$
\begin{gathered}
b_{i}=\alpha_{i}^{\prime-1} f_{\mid U_{i}}^{-1} \alpha_{i}: \\
\mathcal{O}_{X}\left(U_{i}\right) \otimes A \stackrel{\alpha_{i}}{\longrightarrow} \mathcal{O}_{X_{A}}\left(U_{i}\right) \stackrel{f_{\mid U_{i}}^{-1}}{\longrightarrow} \mathcal{O}_{X^{\prime}{ }_{A}}\left(U_{i}\right) \stackrel{\alpha_{i}^{\prime-1}}{\longrightarrow} \mathcal{O}_{X}\left(U_{i}\right) \otimes A .
\end{gathered}
$$

Therefore, $\alpha_{i}^{\prime} b_{i}=f_{\mid U_{i}}^{-1} \alpha_{i}$ and so on $U_{i j}$ we have

this implies

$$
\left(\alpha_{i}^{\prime} b_{i}\right)^{-1}\left(\alpha_{j}^{\prime} b_{j}\right)_{\mid U_{i j}}=\alpha_{i}^{-1} f_{\mid U_{i j}} f_{\mid U_{i j}}^{-1} \alpha_{j}=\alpha_{i}^{-1} \alpha_{j \mid U_{i j}} ;
$$

$$
b_{i}{ }^{-1} \theta_{j i}^{\prime} b_{j}=\theta_{j i} \quad \text { i.e., } \quad \theta_{i j}=b_{j}{ }^{-1} \theta_{i j}^{\prime} b_{i},
$$


where $\theta_{i j}=\alpha_{j}{ }^{-1} \alpha_{i}$ and $\theta_{i j}^{\prime}=\alpha_{j}^{\prime-1} \alpha_{i}^{\prime}$ are the automorphisms of $\mathcal{O}_{X}\left(U_{i j}\right) \otimes A$ corresponding to the deformations $X_{A}$ and $X_{A}^{\prime}$, respectively.

As regards the sheaves, $\phi_{U_{i}}: \mathcal{F}_{A \mid U_{i}} \rightarrow f^{*} \mathcal{F}_{A \mid U_{i}}^{\prime}$ is an isomorphism of $\mathcal{O}_{x_{A}}\left(U_{i}\right)$-modules, for any $i$. The structure of $\mathcal{O}_{X_{A}}$-modules on $\mathcal{F}_{A \mid U_{i}}$ is given by $\alpha_{i}$, while the structure on $f^{*} \mathcal{F}_{A \mid U_{i}}^{\prime}$ si given by $s \cdot x=\alpha_{i}^{\prime-1} f^{-1}(s) x$, for every $s \in \mathcal{O}_{X_{A}}\left(U_{i}\right)$. Therefore, $\phi_{U_{i}}$ has to satisfy $\phi_{U_{i}}(s x)=s \phi_{U_{i}}(x)$, for all $s \in \mathcal{O}_{x_{A}}\left(U_{i}\right)$, where

$$
\phi_{U_{i}}(s x)=\phi_{U_{i}}\left(\alpha_{i}{ }^{-1}(s) x\right) \quad \text { and } \quad s \phi_{U_{i}}(x)=\alpha_{i}^{\prime-1} f^{-1}(s) \phi_{U_{i}}(x) .
$$

Since $\alpha_{i}$ is an isomorphism, there exists $t \in \mathcal{O}_{X}\left(U_{i}\right) \otimes A$ such that $s=\alpha_{i}(t)$. Therefore, we have

$$
\phi_{U_{i}}(t x)=\alpha_{i}^{\prime-1}\left(f^{-1}\left(\alpha_{i}(t)\right)\right) \phi_{U_{i}}(x)=b_{i}(t) \phi_{U_{i}}(x),
$$

for any $t \in \mathcal{O}_{X}\left(U_{i}\right) \otimes A$ and $x \in \mathcal{F}_{\mid U_{i j}} \otimes A$. Moreover, let $\psi_{i j} \in \operatorname{Hom}_{\mathbb{K}}\left(\mathcal{F}_{\mid U_{i j}} \otimes A, \mathcal{F}_{\mid U_{i j}} \otimes A\right)$ and $\psi_{i j}^{\prime} \in \operatorname{Hom}_{\mathbb{K}}\left(\mathcal{F}_{\mid U_{i j}} \otimes A, \mathcal{F}_{\mid U_{i j}} \otimes A\right)$ the automorphism of $\mathcal{O}_{X_{A}}\left(U_{i j}\right)$-modules corresponding to the deformations $\mathcal{F}_{A}$ and $\mathcal{F}_{A}^{\prime}$, respectively; then, the following diagram has to commute:

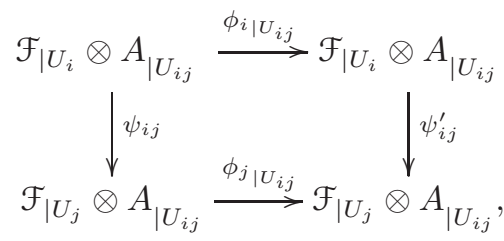

i.e., $\psi_{i j}^{\prime} \phi_{i \mid U_{i j}}=\phi_{j \mid U_{i j}} \psi_{i j}$ that is $\phi_{j \mid U_{i j}}^{-1} \psi_{i j}^{\prime} \phi_{i \mid U_{i j}}=\psi_{i j}$

In conclusion, let $\left(X_{A}, \mathcal{F}_{A}\right)$ and $\left(X_{A}^{\prime}, \mathcal{F}_{A}^{\prime}\right)$ be two locally trivial isomorphic deformations of the pair $(X, \mathcal{F})$ over $A$, corresponding to the elements $\left\{\left(\theta_{i j}, \psi_{i j}\right)\right\}$ and $\left\{\left(\theta_{i j}^{\prime}, \psi_{i j}^{\prime}\right)\right\}$. The isomorphism given by $f: X_{A} \rightarrow X_{A}^{\prime}$ and $\phi: \mathcal{F}_{A} \rightarrow f^{*} \mathcal{F}_{A}^{\prime}$ corresponds to the elements $\left(b_{i}, \phi_{i}\right) \in \mathcal{D}_{A}\left(\mathcal{O}_{X}\left(U_{i}\right) \otimes A, \mathcal{F}\left(U_{i}\right) \otimes A\right)$, for any $i$, such that $b_{i}{ }^{-1} \theta_{i j}^{\prime} b_{j}=\theta_{i j}$ and $\phi_{i}{ }^{-1} \psi_{i j}^{\prime} \phi_{j}=$ $\psi_{i j}$, for any $i$ and $j$.

Since we are in characteristic zero, according to Lemma 2.10, we can take the logarithms and write $\left(b_{i}, \phi_{i}\right)=e^{a_{i}}$, where $a_{i} \in \mathcal{D}_{\mathbb{K}}(X, \mathcal{F})\left(U_{i}\right) \otimes \mathfrak{m}_{A}$. The condition of gluing is equivalent to

$$
e^{-a_{i}} e^{x_{i j}} e^{a_{j}}=e^{x_{i j}^{\prime}}
$$

where $\left\{e^{x_{i j}}=\left(\theta_{i j}, \psi_{i j}\right)\right\}$ and $\left\{e^{x_{i j}^{\prime}}=\left(\theta_{i j}^{\prime}, \psi_{i j}^{\prime}\right)\right\}$.

Theorem 7.5. Let $\mathcal{F}$ be a coherent sheaf on a scheme $X$ of finite type over the field $\mathbb{K}$. Then, the coherent sheaf of Lie algebras $\mathcal{D}_{\mathbb{K}}(X, \mathcal{F})$ controls the problem of locally trivial infinitesimal deformations of the pair $(X, \mathcal{F})$.

Proof. According to Definition 6.6, we have to prove that for any affine open cover $\mathcal{U}=\left\{U_{i}\right\}$ of $X$, there exists an isomorphism of functors of Artin rings

$$
\operatorname{Def}_{\operatorname{Tot}\left(U, \mathcal{D}_{\mathbb{K}}(X, \mathcal{F})\right)} \rightarrow \operatorname{Def}_{(X, \mathcal{F})}^{\mathrm{lt}} .
$$

This follows from the previous consideration and from the explicit description of the functor $\operatorname{Def}_{\operatorname{Tot}\left(\mathcal{L}, \mathcal{D}_{\mathbb{K}}(X, \mathcal{F})\right)}$ given in Remark 6.4: for every $A \in \mathbf{A r t}_{\mathbb{K}}$, we have

$$
\operatorname{Def}_{\operatorname{Tot}\left(u, \mathcal{D}_{\mathbb{K}}(X, \mathcal{F})\right)}(A)=\frac{\left\{\left\{x_{i j}\right\} \in \prod_{i, j} \mathcal{D}_{\mathbb{K}}(X, \mathcal{F})\left(U_{i j}\right) \otimes \mathfrak{m}_{A} \mid e^{x_{j k}} e^{-x_{i k}} e^{x_{i j}}=1\right\}}{\sim},
$$

where $x \sim y$ if and only if there exists $\left\{a_{i}\right\} \in \prod_{i} \mathcal{D}_{\mathbb{K}}(X, \mathcal{F})\left(U_{i}\right) \otimes \mathfrak{m}_{A}$, such that $e^{-a_{i \mid U_{i j}}} e^{x_{i j}} e^{a_{j} \mid U_{i j}}=$ $e^{y_{i j}}$.

Example 7.6. Let $Z$ be a closed subscheme of an algebraic scheme $X$ and denote by $\Theta_{X}(-\log Z) \subset \Theta_{X}$ be the subsheaf of tangent vector fields that are tangent to $Z$. It is known that the sheaf of Lie algebras $\Theta_{X}(-\log Z)$ controls the locally trivial infinitesimal 
deformations of the pair $(X, Z)[14]$. By the same argument used at the beginning of Section 3 , we have two morphisms of sheaves of Lie algebras

$$
\Theta_{X}(-\log Z) \stackrel{\Delta}{\longrightarrow} \mathcal{D}_{\mathbb{K}}\left(X, \mathcal{O}_{Z}\right) \stackrel{\alpha}{\longrightarrow} \Theta_{X}(-\log Z), \quad \alpha \circ \Delta=\mathrm{Id} .
$$

Indeed, the image of the anchor map $\alpha$ preserves the annihilator of the sheaf $\mathcal{O}_{Z}$ and conversely every derivation $h$ preserving the ideal sheaf of $Z$ induces a derivation of pair $(h, h)$.

The geometrical interpretation of these morphisms is clear: the morphism $\Delta$ controls the natural transformation that associates with a locally trivial deformation of $(X, Z)$ the corresponding locally trivial deformation of $\left(X, \mathcal{O}_{Z}\right)$. The anchor map $\alpha$ associates with a locally trivial deformation of the pair $\left(X, \mathcal{O}_{Z}\right)$ the corresponding locally trivial deformation of $\left(X, \operatorname{Supp} \mathcal{O}_{Z}\right)$.

7.2. Infinitesimal deformations. Next, we analyse all the infinitesimal deformations of a pair $(X, \mathcal{F})$. We assume that $X$ is smooth and projective, so that every infinitesimal deformation of $X$ is locally trivial and every coherent sheaf on $X$ has a finite locally free resolution.

Let $\mathcal{E}^{*} \rightarrow \mathcal{F}$ be a finite locally free resolution of $\mathcal{F}$, i.e., an exact sequence

$$
0 \rightarrow \mathcal{E}^{-m} \stackrel{d}{\rightarrow} \mathcal{E}^{-m+1} \stackrel{d}{\rightarrow} \cdots \stackrel{d}{\rightarrow} \mathcal{E}^{-1} \stackrel{d}{\rightarrow} \mathcal{E}^{0} \rightarrow \mathcal{F} \rightarrow 0,
$$

where $\mathcal{E}^{j}$ is a locally free sheaf, for any $j$. According to Example 5.5, we can consider the sheaf of DG-Lie algebras $\mathcal{D}_{\mathbb{K}}^{*}\left(X, \mathcal{E}^{*}\right)$ associated with the complex $\mathcal{E}^{*}$.

Lemma 7.7. Let $\mathcal{F}$ be a coherent sheaf on a smooth projective variety $X$ and $f: \mathcal{E}^{*} \rightarrow \mathcal{F}$ and $g: \mathcal{G}^{*} \rightarrow \mathcal{F}$ two finite locally free resolutions of $\mathcal{F}$. Then, for any open affine cover $\mathcal{U}=\left\{U_{i}\right\}$ of $X$, the $D G$-Lie algebras $\operatorname{Tot}\left(\mathcal{U}, \mathcal{D}_{\mathbb{K}}^{*}\left(X, \mathcal{E}^{*}\right)\right)$ and $\operatorname{Tot}\left(\mathcal{U}, \mathcal{D}_{\mathbb{K}}^{*}\left(X, \mathcal{G}^{*}\right)\right)$ are quasi-isomorphic.

Proof. Since the variety is smooth and projective, by Hilbert Syzygy Theorem, there exist two bounded complexes $\mathcal{H}^{*}$ and $\mathcal{N}^{*}$ of locally free sheaves and a commutative diagram of complexes

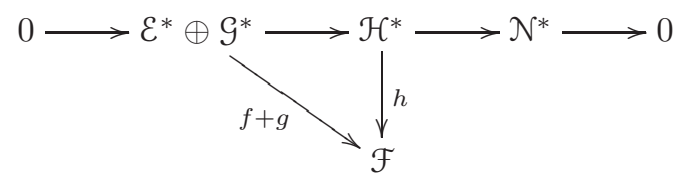

such that $h: \mathcal{H}^{*} \rightarrow \mathcal{F}$ is a quasi-isomorphism; in particular, the induced morphisms $\mathcal{E}^{*} \rightarrow$ $\mathcal{H}^{*}$ and $\mathcal{G}^{*} \rightarrow \mathcal{H}^{*}$ are quasi-isomorphisms.

Let $U$ be an affine open subset of $X$. Since the sections of a locally free sheaf of $\mathcal{O}_{X}$-module over $U$ is a projective $\mathcal{O}_{X}(U)$-module, the injection $\mathcal{E}^{*}(U) \oplus \mathcal{G}^{*}(U) \rightarrow \mathcal{H}^{*}(U)$ is a cofibration, since $\mathcal{H}^{*}, \mathcal{N}^{*}$ are a bounded complexes and therefore $\mathcal{H}^{*}(U), \mathcal{N}^{*}(U)$ are cofibrant complexes of $\mathcal{O}_{X}(U)$-modules. Since $U$ is affine the maps $\mathcal{E}^{*}(U) \oplus \mathcal{G}^{*}(U) \rightarrow \mathcal{F}(U)$ and $\mathcal{H}^{*}(U) \rightarrow \mathcal{F}(U)$ are quasi-isomorphisms and then the induced maps $\mathcal{E}^{*}(U) \rightarrow \mathcal{H}^{*}(U)$ and $\mathcal{G}^{*}(U) \rightarrow \mathcal{H}^{*}(U)$ are trivial cofibrations.

Then, we have the following diagram of morphisms of coherent sheaves of DG-Lie algebras

$$
\mathcal{D}_{\mathbb{K}}^{*}\left(X, \mathcal{E}^{*}\right) \leftarrow \mathcal{D}_{\mathbb{K}}^{*}\left(X, \mathcal{E}^{*} \stackrel{f}{\rightarrow} \mathcal{H}^{*}\right) \rightarrow \mathcal{D}_{\mathbb{K}}^{*}\left(X, \mathcal{H}^{*}\right) \leftarrow \mathcal{D}_{\mathbb{K}}^{*}\left(X, \mathcal{G}^{*} \stackrel{g}{\rightarrow} \mathcal{H}^{*}\right) \rightarrow \mathcal{D}_{\mathbb{K}}^{*}\left(X, \mathcal{G}^{*}\right)
$$


According to Propositions 5.1 and 3.4, for every open $U_{i} \in \mathcal{U}$, we have a diagram of quasiisomorphisms of DG-Lie algebras

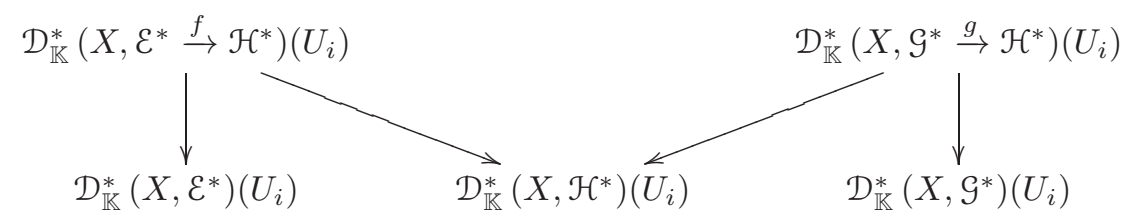

Finally, applying the Tot functor, we conclude that $\operatorname{Tot}\left(\mathcal{U}, \mathcal{D}_{\mathbb{K}}^{*}\left(X, \mathcal{E}^{*}\right)\right)$ and $\operatorname{Tot}\left(\mathcal{U}, \mathcal{D}_{\mathbb{K}}^{*}\left(X, \mathcal{G}^{*}\right)\right)$ are quasi-isomorphic DG-Lie algebras.

Definition 7.8. Let $\mathcal{F}$ be a coherent sheaf on a smooth projective variety $X$. For any $i \in \mathbb{Z}$, we define the coherent sheaves $\mathcal{T}_{(X, \mathcal{F})}^{i}$ as the cohomology sheaves of $\mathcal{D}_{\mathbb{K}}^{*}\left(X, \mathcal{E}^{*}\right)$, for any finite locally free resolution $\mathcal{E}^{*} \rightarrow \mathcal{F}$ of $\mathcal{F}$ :

$$
\mathcal{T}_{(X, \mathcal{F})}^{i}:=\mathcal{H}^{i}\left(\mathcal{D}_{\mathbb{K}}^{*}\left(X, \mathcal{E}^{*}\right)\right) .
$$

Similarly, we define the hyper-cohomology groups $T_{(X, \mathcal{F})}^{i}=\mathbb{H}^{i}\left(X, \mathcal{D}_{\mathbb{K}}^{*}\left(X, \mathcal{E}^{*}\right)\right)$. Lemma 7.7 implies that the sheaves $\mathcal{T}_{(X, \mathcal{F})}^{i}$ and the groups $T_{(X, \mathcal{F})}^{i}$ are well defined, since they do not depend on the choice of the resolution.

The short exact sequence (5.3) of complexes of coherent sheaves

$$
0 \rightarrow \mathcal{H}_{0} \mathrm{O}_{X}\left(\mathcal{E}^{*}, \mathcal{E}^{*}\right) \rightarrow \mathcal{D}_{\mathbb{K}}^{*}\left(X, \mathcal{E}^{*}\right) \rightarrow \Theta_{X} \rightarrow 0
$$

gives a hypercohomology long exact sequence

$$
0 \rightarrow \operatorname{Ext}_{X}^{0}(\mathcal{F}, \mathcal{F}) \rightarrow T_{(X, \mathcal{F})}^{0} \rightarrow H^{0}\left(X, \Theta_{X}\right) \rightarrow \operatorname{Ext}_{X}^{0}(\mathcal{F}, \mathcal{F}) \rightarrow T_{(X, \mathcal{F})}^{1} \rightarrow \cdots
$$

By the hypercohomology spectral sequence, we have $E_{2}^{p, q}=H^{p}\left(X, \mathcal{T}_{(X, \mathcal{F})}^{q}\right) \Rightarrow T_{(X, \mathcal{F})}^{p+q}$. According to Example 3.6, we have that $\mathcal{T}_{(X, \mathcal{F})}^{i}=\mathcal{E} x t_{\mathcal{O}_{X}}^{i}(\mathcal{F}, \mathcal{F})$, for $i \neq 0,1$, and we have an exact sequence of sheaves

$$
0 \rightarrow \mathcal{H}^{\operatorname{lom}_{\mathcal{O}_{X}}}(\mathcal{F}, \mathcal{F}) \rightarrow \mathcal{T}_{(X, \mathcal{F})}^{0} \rightarrow \Theta_{X} \rightarrow \mathcal{E x}_{\mathcal{O}_{X}}^{1}(\mathcal{F}, \mathcal{F}) \rightarrow \mathcal{T}_{(X, \mathcal{F})}^{1} \rightarrow 0 .
$$

In particular, the sheaf $\mathcal{T}_{(X, \mathcal{F})}^{i}$ vanishes in the locus where $\mathcal{F}$ has projective dimension $<i$.

Local Case. First of all, we analyse the infinitesimal deformations of the pair $(X, \mathcal{F})$ in the local case. Let $X=\operatorname{Spec} R$, where $R$ is smooth and affine over $\mathbb{K}$ and $\mathcal{F}=\widetilde{M}$, where $M$ is a finitely generated $R$-module. Let $\left(E^{*}, d\right)$ be a finite complex of projective $R$-module, such that the sequence

$$
0 \rightarrow E^{-m} \stackrel{d}{\rightarrow} E^{-m+1} \stackrel{d}{\rightarrow} \cdots \stackrel{d}{\rightarrow} E^{-1} \stackrel{d}{\rightarrow} E^{0} \rightarrow M \rightarrow 0,
$$

is exact. Finally, consider the DG-Lie algebra $D_{\mathbb{K}}^{*}\left(R, E^{*}\right)$.

Proposition 7.9. In the notation above, the DG-Lie algebra $D_{\mathbb{K}}^{*}\left(R, E^{*}\right)$ controls the infinitesimal deformations of the pair $(X, \mathcal{F})$, i.e., there exists an isomorphism of deformation functors

$$
\operatorname{Def}_{D_{\mathbb{K}}^{*}\left(R, E^{*}\right)} \cong \operatorname{Def}_{(X, \mathcal{F})} \text {. }
$$

Proof. The variety $X=\operatorname{Spec} R$ is smooth and affine and so it has only trivial infinitesimal deformations [25, Theorem 1.2.4]. Therefore, for any $A \in \mathbf{A r t}_{\mathbb{K}}$, any infinitesimal deformation of the pair $(X, \mathcal{F}=\widetilde{M})$ over $A$ is of the form $\left(X \times \operatorname{Spec} A, F_{A}=\widetilde{M}_{A}\right)$, where $M_{A}$ is an $R \otimes A$-module, that is $A$-flat, together with a morphism $\pi: M_{A} \rightarrow M$, inducing an isomorphism $M_{A} \otimes_{A} \mathbb{K} \rightarrow M[6$, Section 1].

The flatness condition allows to lift the relations; therefore any deformation $M_{A}$ of $M$ over $A$ corresponds to an exact sequence $E^{*} \otimes A \rightarrow M_{A}$ that reduces to $E^{*} \rightarrow M$, when tensored 
with $\mathbb{K}$, i.e., any deformation $M_{A}$ of $M$ over $A$ corresponds to a deformed complex $\left(E^{*} \otimes\right.$ $\left.A, d_{A}\right)$ and any of these complexes is of the form $\left(E^{*} \otimes A, d+x\right)$, for $x \in \operatorname{MC}_{D_{\mathbb{K}}^{*}\left(R, E^{*}\right)}(A)=$ $\mathrm{MC}_{\mathrm{Hom}_{\mathbb{K}}^{*}\left(E^{*}, E^{*}\right)}(A)$.

Two deformations $\left(X \times \operatorname{Spec} A, \mathcal{F}_{A}=\widetilde{M}_{A}\right)$ and $\left(X \times \operatorname{Spec} A, \mathcal{F}_{A}^{\prime}=\widetilde{M}_{A}^{\prime}\right)$ of $(X, \widetilde{M})$ over $A$ are isomorphic if there exist an isomorphism of deformations $f: X \times \operatorname{Spec} A \rightarrow X \times \operatorname{Spec} A$ and an isomorphism $\mathcal{F}_{A} \rightarrow f^{*} \mathcal{F}_{A}^{\prime}$ of $R \otimes A$-modules.

In particular, the isomorphism of the modules lifts to an isomorphism of the deformed complexes and vice versa. Then, an isomorphism is given by a pair $(\theta, \psi)$ where $\theta: R \otimes A \rightarrow$ $R \otimes A$ is an isomorphism that lifts the identity of $R$ and $\psi:\left(E^{*} \otimes A, d+x\right) \rightarrow\left(E^{*} \otimes A, d+x^{\prime}\right)$ is an isomorphism, lifting the identity, such that $\psi(r m)=\theta(r) \psi(m)$, for any $r \in R \otimes A$ and any $m \in E^{*} \otimes A$.

As in Lemma 2.10, for any $A \in \mathbf{A r t}_{\mathbb{K}}$ the group $\exp \left(D_{\mathbb{K}}^{0}\left(R, E^{*}\right) \otimes \mathfrak{m}_{A}\right)$ is naturally isomorphic to the group of $A$-automorphisms of the pair $\left(R \otimes A, E^{*} \otimes A\right)$ lifting the identity on $\left(R, E^{*}\right)$; therefore, it corresponds to the group of the isomorphisms of deformations.

Example 7.10 (cf. [5, Proposition A3]). Assume that $X$ is a smooth surface, $D \subset X$ a reduced divisor and $\mathcal{F}$ a sheaf of $\mathcal{O}_{D}$-module. Assume that for every point $p \in D$, the stalk $\mathcal{F}_{p}$ is a torsion free $\mathcal{O}_{D, p}$-module. Then, by Auslander-Buchsbaum Theorem $[21$, Theorem 19.1], the projective dimension of every stalk of the sheaf $\mathcal{F}$ is at most 1 . In particular, $\mathcal{T}_{(X, \mathcal{F})}^{i}=\mathcal{E} x t_{\mathcal{O}_{X}}^{i}(\mathcal{F}, \mathcal{F})=0$, for every $i \geq 2$.

If $X$ is affine, we have $\operatorname{Ext}_{X}^{2}(\mathcal{F}, \mathcal{F})=H^{0}\left(\mathcal{E} x t_{\mathcal{O}_{X}}^{2}(\mathcal{F}, \mathcal{F})\right)=0$, and so the pair $(X, \mathcal{F})$ has unobstructed deformations.

Global Case. Finally, we analyse all the infinitesimal deformations of a pair $(X, \mathcal{F})$. Essentially, we have to glue together the computations about the locally trivial infinitesimal deformations (Theorem 7.5) and the deformations in the local case (Proposition 7.9).

Theorem 7.11. Let $\mathcal{F}$ be a coherent sheaf on a projective smooth variety $X$ and $\mathcal{E}^{*} \rightarrow \mathcal{F}$ a finite locally free resolution of $\mathcal{F}$. Then, the coherent sheaf of DG-Lie algebras $\mathcal{D}_{\mathbb{K}}^{*}\left(X, \mathcal{E}^{*}\right)$ controls the problem of deformations of the pair $(X, \mathcal{F})$. In particular, $T_{(X, \mathcal{F})}^{1}$ is the tangent space and $T_{(X, \mathcal{F})}^{2}$ is the obstructions space for $\operatorname{Def}_{(X, \mathcal{F})}$.

Proof. The proof follows the general lines already used in [6]. According to Definition 6.6, we have to prove that for any affine open cover $\mathcal{U}=\left\{U_{i}\right\}$ of $X$, there exists an isomorphism of functors of Artin rings

$$
\operatorname{Def}_{\operatorname{Tot}\left(U, \mathcal{D}_{\mathbb{K}}^{*}\left(X, \mathcal{E}^{*}\right)\right)} \rightarrow \operatorname{Def}_{(X, \mathcal{F})} .
$$

According to the isomorphism given in Equation (6.1), it is enough to show that there exists an isomorphism $H_{\mathrm{sc}}^{1}\left(\exp \left(\mathcal{D}_{\mathbb{K}}^{*}\left(X, \mathcal{E}^{*}\right)(\mathcal{U})\right)\right) \rightarrow \operatorname{Def}_{(X, \mathcal{F})}$. Therefore, for any $A \in \mathbf{A r t}_{\mathbb{K}}$, we have to prove that every element in $Z_{\mathrm{sc}}^{1}\left(\exp \left(\mathcal{D}_{\mathbb{K}}^{*}\left(X, \mathcal{E}^{*}\right)(\mathcal{U})\right)\right)(A)$ corresponds to an infinitesimal deformation of the pair $(X, \mathcal{F})$ over $A$ and that two elements are equivalent if and only if the corresponding deformations are isomorphic. This follows from the local study analysed in Proposition 7.9 and a gluing procedure as in Theorem 7.5. Indeed, an element $(l, m) \in Z_{\mathrm{sc}}^{1}\left(\exp \left(\mathcal{D}_{\mathbb{K}}^{*}\left(X, \mathcal{E}^{*}\right)(\mathcal{U})\right)\right)(A)$ gives a deformation of the pair $(X, \mathcal{F})$ as gluing of deformations on each $U_{i}$. We only stress the fact that the gluing condition on the isomorphisms involves an element in $\prod_{i, j, k} \mathcal{H} m_{\mathcal{O}_{X}}^{-1}\left(\mathcal{E}^{*}, \mathcal{E}^{*}\right)\left(U_{i j k}\right) \otimes \mathfrak{m}_{A}$; this is due to the fact that we do not have to glue the deformed complexes but rather their cohomology to get a sheaf (see [6, Section 2] for more details about this). As regards the equivalence relation $\sim$, the first condition is the isomorphism of the induced deformation on each open, the second condition gives the gluing of the local isomorphism to have a global isomorphism of the induced deformations. 
More explicitly, an element $(l, m) \in Z_{\mathrm{sc}}^{1}\left(\exp \left(\mathcal{D}_{\mathbb{K}}^{*}\left(X, \mathcal{E}^{*}\right)(\mathcal{U})\right)\right)(A)$ is given by the sequences $l=\left\{l_{i}\right\} \in \prod_{i} \mathcal{H}_{o} m_{\mathcal{O}_{X}}^{1}\left(\mathcal{E}^{*}, \mathcal{E}^{*}\right)\left(U_{i}\right) \otimes \mathfrak{m}_{A}$ and $m=\left\{\left(h_{i j}, u_{i j}\right)\right\} \in \prod_{i, j} \mathcal{D}_{\mathbb{K}}^{0}\left(X, \mathcal{E}^{*}\right)\left(U_{i j}\right) \otimes$ $\mathfrak{m}_{A}$ such that:

(1) for any $i$, the element $l_{i}$ satisfies the Maurer-Cartan equation, i.e., $d l_{i}+\frac{1}{2}\left[l_{i}, l_{i}\right]=0$;

(2) for any $i$ and $j$, the restriction of $l_{i}$ and $l_{j}$ to $U_{i j}$ are gauge equivalent under $m_{i j}$, i.e., $\left.l_{i}\right|_{U_{i j}}=\left.e^{m_{i j}} * l_{j}\right|_{U_{i j}}$.

(3) for any $i, j$ and $k$, we have the following conditions:

$$
\begin{gathered}
\left.h_{j k}\right|_{U_{i j k}} \bullet-\left.\left.h_{i k}\right|_{U_{i j k}} \bullet h_{i j}\right|_{U_{i j k}}=0 \\
\left.u_{j k}\right|_{U_{i j k}} \bullet-\left.\left.u_{i k}\right|_{U_{i j k}} \bullet u_{i j}\right|_{U_{i j k}}=\left[d+\left.l_{j}\right|_{U_{i j k}}, n_{i j k}\right], \\
\text { for some } n=\left\{n_{i j k}\right\} \in \prod_{i, j, k} \mathcal{H o m}_{\mathcal{O}_{X}}^{-1}\left(\mathcal{E}^{*}, \mathcal{E}^{*}\right)\left(U_{i j k}\right) \otimes \mathfrak{m}_{A} .
\end{gathered}
$$

According to the local study, condition (1) and (2) imply that every $l_{i}$ defines a deformation of the pair $(X, \mathcal{F})$ on $U_{i}$ and that the deformations induced by $l_{i}$ and $l_{j}$ on $U_{i j}$ are isomorphic. Finally, the third condition implies that we can glue together the local deformations to get a global deformation the pair $(X, \mathcal{F})$ over $A$. Indeed, the former condition is the gluing of the locally trivial deformation of $U_{i}$ over the double intersections to have a deformation of $X$ over $A$; the latter regards the gluing of the local deformations of the sheaf $\mathcal{F}$. We only stress the fact that the isomorphisms that glues the restriction of the deformed complexes $\left(\mathcal{E}_{U_{i}}^{*} \otimes A, d+l_{i}\right)$ and $\left(\mathcal{E}_{U_{j}}^{*} \otimes A, d+l_{j}\right)$, for any $i$ and $j$ satisfy the cocycle condition only up to homotopy. Indeed, we do not have to glue the restriction of the deformed complexes of sheaves together but rather their cohomology. Therefore, the gluing isomorphism have to satisfy the cocycle condition only up to homotopy.

As regard the equivalence relation, suppose that $\left(l_{0}=\left\{l_{0, i}\right\}, m_{0}=\left\{\left(h_{0, i j}, u_{0, i j}\right)\right\}\right)$ and $\left(l_{1}=\left\{l_{1, i}\right\}, m_{1}=\left\{\left(h_{1, i j}, u_{1, i j}\right)\right\}\right) \in Z_{\mathrm{sc}}^{1}\left(\exp \left(\mathcal{D}_{\mathbb{K}}^{*}\left(X, \mathcal{E}^{*}\right)(\mathcal{U})\right)\right)(A)$ are equivalent under the relation $\sim$, then we need to prove that the induced deformations are isomorphic. The equivalence implies the existence of $a=\left\{g_{i}, v_{i}\right\} \in \prod_{i} \mathcal{D}_{\mathbb{K}}^{0}\left(X, \mathcal{E}^{*}\right)\left(U_{i}\right) \otimes \mathfrak{m}_{A}$ and $b=\left\{b_{i j}\right\} \in$ $\prod_{i, j} \mathcal{H}_{0} m_{\mathcal{O}_{X}}^{-1}\left(\mathcal{E}^{*}, \mathcal{E}^{*}\right)\left(U_{i j}\right) \otimes \mathfrak{m}_{A}$ such that

$$
\left\{\begin{array}{l}
e^{a} * l_{0}=l_{1} \\
-m_{0} \bullet-a_{i \mid U_{i j}} \bullet m_{1} \bullet a_{j \mid U_{i j}}=d b+\left[l_{0, j \mid U_{i j}}, b\right] .
\end{array}\right.
$$

The first condition implies that the deformations of the pair induced by $l_{0}$ and $l_{1}$ are isomorphic locally on $U_{i}$; the second condition provides the gluing of the isomorphisms along double intersections. (We have a strict cocycle condition for the isomorphisms of the deformations of $X$ and, as before, we have a homotopy cocycle condition for the gluing of the deformed complexes.)

As regard the tangent and obstruction space for $\operatorname{Def}_{(X, \mathcal{F})}$, we have that $H^{1}\left(\operatorname{Tot}\left(\mathcal{U}, \mathcal{D}_{\mathbb{K}}^{*}\left(X, \mathcal{E}^{*}\right)\right)\right)$ is the tangent space and $H^{2}\left(\operatorname{Tot}\left(\mathcal{U}, \mathcal{D}_{\mathbb{K}}^{*}\left(X, \mathcal{E}^{*}\right)\right)\right)$ is an obstruction space. According to Definition 7.8 and to Equality (6.2), we have that

$$
H^{i}\left(\operatorname{Tot}\left(\mathcal{D}_{\mathbb{K}}^{*}\left(X, \mathcal{E}^{*}\right)(\mathcal{U})\right)\right) \cong \mathbb{H}^{i}\left(X, \mathcal{D}_{\mathbb{K}}^{*}\left(X, \mathcal{E}^{*}\right)\right)=T_{(X, \mathcal{F})}^{i} .
$$

Remark 7.12. In the situation of Theorem 7.11, consider the sheaf of truncated DG-Lie algebras

$$
\sigma_{\leq 0} \mathcal{D}_{\mathbb{K}}^{*}\left(X, \mathcal{E}^{*}\right)=\mathcal{D}_{\mathbb{K}}\left(X, \mathcal{E}^{*}\right) \oplus_{i<0} \mathcal{H}_{0} m_{\mathcal{O}_{X}}^{i}\left(\mathcal{E}^{*}, \mathcal{E}^{*}\right) \subset \mathcal{D}_{\mathbb{K}}^{*}\left(X, \mathcal{E}^{*}\right)
$$

We have seen in Example 3.6, that the natural map $\mathcal{D}_{\mathbb{K}}\left(X, \mathcal{E}^{*}\right) \rightarrow \mathcal{D}_{\mathbb{K}}(X, \mathcal{F})$ induces a surjective quasi-isomorphism of coherent sheaves of DG-Lie algebras $\sigma_{\leq 0} \mathcal{D}_{\mathbb{K}}^{*}\left(X, \mathcal{E}^{*}\right) \rightarrow$ $\mathcal{D}_{\mathbb{K}}(X, \mathcal{F})$. Therefore, also the sheaf of truncated DG-Lie algebras $\sigma_{\leq 0} \mathcal{D}_{\mathbb{K}}^{*}\left(X, \mathcal{E}^{*}\right)$ controls the locally trivial deformations of the pair, and the obvious natural transformation $\operatorname{Def}_{(X, \mathcal{F})}^{\mathrm{lt}} \rightarrow \operatorname{Def}_{(X, \mathcal{F})}$ is induced by the inclusion $\sigma_{\leq 0} \mathcal{D}_{\mathbb{K}}^{*}\left(X, \mathcal{E}^{*}\right) \subset \mathcal{D}_{\mathbb{K}}^{*}\left(X, \mathcal{E}^{*}\right)$. 
We are now ready to prove the last item of Theorem 1.2.

Proposition 7.13. Let $\mathcal{F}$ be a coherent sheaf on a projective smooth variety $X$. Assume that $H^{0}\left(X, \Theta_{X}\right)=0$ and $\operatorname{Ext}_{X}^{0}(\mathcal{F}, \mathcal{F})=\mathbb{K}$, then the functor $\operatorname{Def}_{(X, \mathcal{F})}$ is pro-representable.

Proof. Let $\mathcal{E}^{*} \rightarrow \mathcal{F}$ be a finite locally free resolution of $\mathcal{F}$. According to (7.1) the vector space $T_{(X, \mathcal{F})}^{0}$ is one-dimensional generated by the identity on the complex $\mathcal{E}^{*}$. Therefore, for every open affine cover $\mathcal{U}$ the vector space $H^{0}\left(\operatorname{Tot}\left(\mathcal{U}, \mathcal{D}_{\mathbb{K}}^{*}\left(X, \mathcal{E}^{*}\right)\right)\right)$ is one-dimensional and generated by the identity on $\mathcal{E}_{\mid U_{i_{0} \cdots i_{k}}}^{*}$ for every $k \geq 0$ and every $i_{0}, \ldots, i_{k}$. In particular the DG-Lie algebra $\operatorname{Tot}\left(\mathcal{U}, \mathcal{D}_{\mathbb{K}}^{*}\left(X, \mathcal{E}^{*}\right)\right)$ satisfies the condition of Lemma 6.2 and the conclusion follows by Theorem 7.11 .

Let $X$ be a projective smooth variety, $\mathcal{F}$ a coherent sheaf on $X$ and $\mathcal{E}^{*} \rightarrow \mathcal{F}$ a finite locally free resolution. By definition $\operatorname{det} \mathcal{F}=\operatorname{det} \mathcal{E}^{*}$, and we have defined a morphism of sheaves of DG-Lie algebras (see Equation (5.7)):

$$
\operatorname{Tr}: \mathcal{D}_{\mathbb{K}}^{*}\left(X, \mathcal{E}^{*}\right) \rightarrow \mathcal{D}_{\mathbb{K}}(X, \operatorname{det} \mathcal{F}) .
$$

By Theorem 7.11 , the sheaf $\mathcal{D}_{\mathbb{K}}{ }^{*}\left(X, \mathcal{E}^{*}\right)$ controls the deformations of the pair $(X, \mathcal{F})$, while the sheaf $\mathcal{D}_{\mathbb{K}}(X, \operatorname{det} \mathcal{F})$ controls the deformations of the pair $(X, \operatorname{det} \mathcal{F})$.

We can interpret this morphism as a natural transformation of deformation functors. Some deformations of the pair $(X, \mathcal{F})$ corresponds to a deformation of $X$ and of the complex $\mathcal{E}^{*}$; then with these deformations we can easily associate a deformation of the pair $\left(X, \operatorname{det} \mathcal{F}=\operatorname{det} \mathcal{E}^{*}\right)$, considering the determinant of the deformed complex. In general, not all the deformations of $\mathcal{F}$ corresponds to a deformations of the complex, but this is true only locally on $X$ and the deformations of the complex glues only in cohomology. Anyway, we can still define a deformation of the pair $(X, \operatorname{det} \mathcal{F})$, as we view in the following lemma.

Lemma 7.14. Let $X$ be a projective smooth variety and $\mathcal{F}$ a coherent sheaf on $X$. Then, there exists a commutative diagram of deformation functors

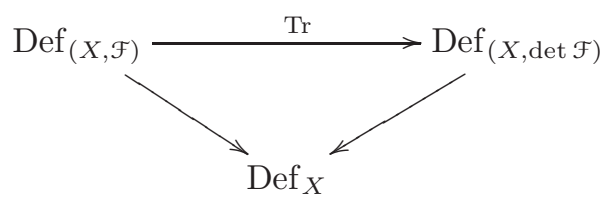

where the diagonal arrows are the forgetful natural transformations, and the natural transformation $\operatorname{Tr}$ is induced by the trace map $\operatorname{Tr}: \mathcal{D}_{\mathbb{K}}^{*}\left(X, \mathcal{E}^{*}\right) \rightarrow \mathcal{D}_{\mathbb{K}}^{*}(X$, $\operatorname{det} \mathcal{F})$, for any finite locally free resolution $\mathcal{E}^{*} \rightarrow \mathcal{F}$.

Proof. Let $\mathcal{U}=\left\{U_{i}\right\}$ be an open affine cover for $X$, such that every sheaf $\mathcal{E}^{k}$ is free on every $U_{i}$. Fix $A \in \operatorname{Art}_{\mathbb{K}}$ and consider a deformation $\left(X_{A}, \mathcal{F}_{A}\right) \in \operatorname{Def}_{(X, \mathcal{F})}(A)$. According to Theorem 7.11, this deformation corresponds to an element $(l, m) \in Z_{\mathrm{sc}}^{1}\left(\exp \left(\mathcal{D}_{\mathbb{K}}^{*}\left(X, \mathcal{E}^{*}\right)(\mathcal{U})\right)\right)(A)$, where $l=\left\{l_{i}\right\} \in \prod_{i} \mathcal{H} \mathcal{H o m}_{\mathcal{O}_{X}}^{1}\left(\mathcal{E}^{*}, \mathcal{E}^{*}\right)\left(U_{i}\right) \otimes \mathfrak{m}_{A}$ and $m=\left\{\left(h_{i j}, u_{i j}\right)\right\} \in \prod_{i, j} \mathcal{D}_{\mathbb{K}}^{0}\left(X, \mathcal{E}^{*}\right)\left(U_{i j}\right) \otimes$ $\mathfrak{m}_{A}$. In particular, for any $i, j$ and $k$, we have the following conditions:

$$
\left.u_{j k}\right|_{U_{i j k}} \bullet-\left.\left.u_{i k}\right|_{U_{i j k}} \bullet u_{i j}\right|_{U_{i j k}}=\left[d+\left.l_{j}\right|_{U_{i j k}}, n_{i j k}\right],
$$

for some $n=\left\{n_{i j k}\right\} \in \prod_{i, j, k} \mathcal{H} m_{\mathcal{O}_{X}}^{-1}\left(\mathcal{E}^{*}, \mathcal{E}^{*}\right)\left(U_{i j k}\right) \otimes \mathfrak{m}_{A}$. Applying the trace morphisms to $m$, for any $i$ and $j$, we have

$$
\operatorname{Tr}\left(h_{i j}, u_{i j}\right)=\left(h_{i j}, \operatorname{Tr}\left(u_{i j}\right)\right) \in \mathcal{D}_{\mathbb{K}}^{0}(X, \operatorname{det} \mathcal{F})\left(U_{i j}\right) \otimes \mathfrak{m}_{A}
$$

such that

$$
\operatorname{Tr}\left(\left.u_{j k}\right|_{U_{i j k}}\right) \bullet-\operatorname{Tr}\left(\left.u_{i k}\right|_{U_{i j k}}\right) \bullet \operatorname{Tr}\left(\left.u_{i j}\right|_{U_{i j k}}\right)=\operatorname{Tr}\left(\left[d+\left.l_{j}\right|_{U_{i j k}}, n_{i j k}\right]\right)=0 .
$$


This implies that the element $\operatorname{Tr}(m)=\left\{\left(h_{i j}, \operatorname{Tr}\left(u_{i j}\right)\right\} \in \prod_{i, j} \mathcal{D}_{\mathbb{K}}^{0}(X, \operatorname{det} \mathcal{F})\left(U_{i j}\right) \otimes \mathfrak{m}_{A}\right.$ satisfies the cocycle condition on triple intersections and so, by Theorem 7.5, it defines a deformation of the pair $(X, \operatorname{det} \mathcal{F})$ over $A$.

An analogous computation shows that the isomorphism class of the induced deformation of $(X, \operatorname{det} \mathcal{F})$ does not depend on the isomorphism class of the deformation of $(X, \mathcal{F})$. Indeed, let $\left(X_{A}, \mathcal{F}_{A}\right)$ and $\left(X_{A}^{\prime}, \mathcal{F}_{A}^{\prime}\right)$ be isomorphic deformations, corresponding to the elements $\left(l_{0}=\left\{l_{0, i}\right\}, m_{0}=\left\{\left(h_{0, i j}, u_{0, i j}\right)\right\}\right)$ and $\left(l_{1}=\left\{l_{1, i}\right\}, m_{1}=\left\{\left(h_{1, i j}, u_{1, i j}\right)\right\}\right) \in$ $Z_{\mathrm{sc}}^{1}\left(\exp \left(\mathcal{D}_{\mathbb{K}}^{*}\left(X, \mathcal{E}^{*}\right)(\mathcal{U})\right)\right)(A)$. According to Theorem 7.11, the isomorphism corresponds to the existence of

$$
a=\left\{g_{i}, v_{i}\right\} \in \prod_{i} \mathcal{D}_{\mathbb{K}}^{0}\left(X, \mathcal{E}^{*}\right)\left(U_{i}\right) \otimes \mathfrak{m}_{A} \quad \text { and } \quad b=\left\{b_{i j}\right\} \in \prod_{i, j} \mathcal{H}_{0} m_{\mathcal{O}_{X}}^{-1}\left(\mathcal{E}^{*}, \mathcal{E}^{*}\right)\left(U_{i j}\right) \otimes \mathfrak{m}_{A}
$$

such that

$$
\left\{\begin{array}{l}
e^{a} * l_{0}=l_{1} \\
-m_{0} \bullet-a_{i \mid U_{i j}} \bullet m_{1} \bullet a_{j \mid U_{i j}}=d b+\left[l_{0, j \mid U_{i j}}, b\right] .
\end{array}\right.
$$

In particular, applying the trace morphisms for any $i$ and $j$, we have

$$
-\operatorname{Tr}\left(m_{0}\right) \bullet-\operatorname{Tr}\left(a_{i \mid U_{i j}}\right) \bullet \operatorname{Tr}\left(m_{1}\right) \bullet \operatorname{Tr}\left(a_{j} U_{i j}\right)=0 \in \mathcal{D}_{\mathbb{K}}^{0}(X, \operatorname{det} \mathcal{F})\left(U_{i j}\right) \otimes \mathfrak{m}_{A} .
$$

By Theorem 7.5, this implies that the induced deformations of $(X, \operatorname{det} \mathcal{F})$ are isomorphic.

Remark 7.15. As a particular case, if we only consider the deformations of a coherent sheaf $\mathcal{F}$ on a fixed projective variety $X$, then trace induces a morphism of functors

$$
\operatorname{Def}_{\mathcal{F}} \rightarrow \operatorname{Def}_{\operatorname{det} \mathcal{F}} .
$$

In this case, the induced morphisms in cohomology are the ones already analysed in $[27$, Theorem 3.23].

Theorem 7.16. Let $X$ be a projective smooth variety and $\mathcal{E}^{*}$ a locally free finite resolution of a coherent sheaf $\mathcal{F}$. Consider the linear maps $\operatorname{Tr}^{i}$ : $\operatorname{Ext}_{X}^{i}(\mathcal{F}, \mathcal{F}) \rightarrow H^{i}\left(X, \mathcal{O}_{X}\right)$ induced by the morphism of sheaves of DG-Lie algebras $\operatorname{Tr}: \mathcal{H} m_{\mathcal{O}_{X}}^{*}\left(\mathcal{E}^{*}, \mathcal{E}^{*}\right) \rightarrow \mathcal{O}_{X}$.

If the map $\operatorname{Tr}^{1}$ is surjective and the map $\operatorname{Tr}^{2}$ is injective, then the induced natural transformation $\operatorname{Def}_{(X, \mathcal{F})} \rightarrow \operatorname{Def}_{(X, \operatorname{det} F)}$ is smooth. If $\operatorname{Tr}^{2}$ is injective and $\operatorname{Def}(X, \operatorname{det} \mathcal{F})$ is unobstructed, then $\operatorname{Def}_{(X, F)}$ is unobstructed.

By the exact sequence (5.5), the above assumptions on $\operatorname{Tr}^{1}$ and $\operatorname{Tr}^{2}$ are equivalent to assuming $\operatorname{Ext}_{X}^{2}(\mathcal{F}, \mathcal{F})_{0}=0$.

Proof. By Theorem 5.6, there exists a commutative diagram with exact rows and columns of morphisms of coherent sheaves of DG-Lie algebras

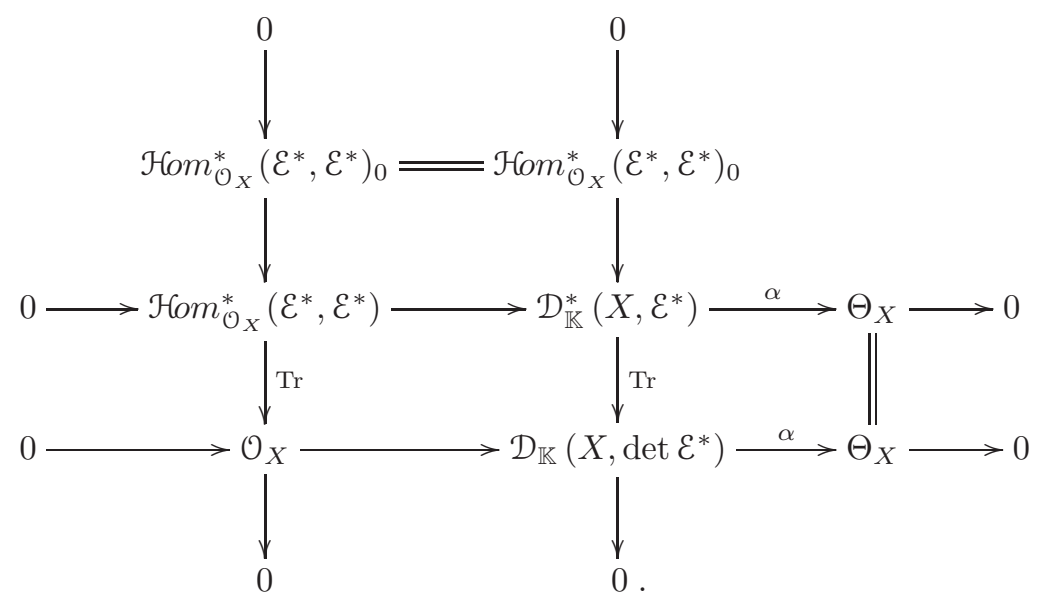


The hypothesis on the morphisms $\operatorname{Tr}: \mathcal{H} m_{\mathcal{O}_{X}}^{*}\left(\mathcal{E}^{*}, \mathcal{E}^{*}\right) \rightarrow \mathcal{O}_{X}$ implies that the same holds for the morphisms induced by $\operatorname{Tr}: \mathcal{D}_{\mathbb{K}}{ }^{*}\left(X, \mathcal{E}^{*}\right) \rightarrow \mathcal{D}_{\mathbb{K}}(X, \operatorname{det} \mathcal{F})$. Then, it is enough to apply the Standard Smoothness Criterion [19, Theorem 4.11] to the corresponding morphism of functors $\operatorname{Tr}: \operatorname{Def}_{(X, \mathcal{F})} \rightarrow \operatorname{Def}_{(X, \operatorname{det} \mathcal{F})}$.

If $\operatorname{Def}_{(X, \operatorname{det} \mathcal{F})}$ is unobstructed, then $\operatorname{Tr}^{2}: T_{(X, \mathcal{F})}^{2} \rightarrow T_{(X, \operatorname{det} \mathcal{F})}^{2}$ annihilates all the obstructions and its injectivity implies that also $\operatorname{Def}_{(X, \mathcal{F})}$ is unobstructed.

Acknowledgement. D.I. wish to thank the Dipartimento di Matematica "G. Castelnuovo" of Sapienza Università di Roma for the hospitality during the preparation of this paper. D.I. acknowledges the support of Fondi di Ateneo dell'Università di Bari; M.M. acknowledges the support by Italian MIUR under PRIN project 2015ZWST2C "Moduli spaces and Lie theory". We wish to thank the referees for useful comments and for suggestions improving the presentation of the paper.

\section{REFERENCES}

[1] I.V. Artamkin: On deformations of sheaves, Math. USSR Izvestiya 32 (1989), No. 3, 663-668. 1

[2] M. Atiyah: Complex analytic connections in fibre bundles, Trans. Am. Math. Soc. 85 (1957), 181-207. 12

[3] R. Bandiera and M. Manetti: On coisotropic deformations of holomorphic submanifolds, J. Math. Sci. Univ. Tokyo 22 (2015), no. 1, 1-37; arXiv:1301.6000. 16

[4] D. Eisenbud: Commutative algebra. With a view toward algebraic geometry, Graduate Texts in Mathematics, 150, Springer-Verlag, New York, (1995). 11

[5] B. Fantechi, L. Göttsche and D. van Straten: Euler number of the compactified Jacobian and multiplicity of rational curves, J. Algebraic Geometry 8 (1999), 115-133; arXiv:alg-geom/9708012. 23

[6] D. Fiorenza, D. Iacono and E. Martinengo: Differential graded Lie algebras controlling infinitesimal deformations of coherent sheaves, J. Eur. Math. Soc. (JEMS) 14 (2012), no. 2, 521-540; arXiv:0904.1301. $2,15,16,17,22,23$

[7] D. Fiorenza, M. Manetti and E. Martinengo: Cosimplicial DGLAs in deformation theory, Communications in Algebra 40 (2012), 2243-2260; arXiv:0803.0399. 16, 17

[8] W.M. Goldman and J.J. Millson: The deformation theory of representations of fundamental groups of compact kähler manifolds, Publ. Math. I.H.E.S. 67 (1988), 43-96. 15

[9] A. Grothendieck: Éléments de Géométrie Algébrique IV, quatrième partie, Publ. Math. IHES no. 32 (1967), 5-361. 2

[10] R. Hart: Derivations on commutative rings, J. London Math. Soc. (2) 8 (1974), 171-175. 11

[11] R. Hartshorne: Algebraic geometry, Graduate Texts in Mathematics, 52, Springer-Verlag, New York, (1977). 12

[12] M. Hovey: Model categories, Mathematical Surveys and Monographs, vol. 63, American Mathematical Society, (1999). 9, 10

[13] L. Huang: On joint moduli spaces, Math. Ann. 302 (1995), 61-79. 2

[14] D. Iacono: Deformations and obstructions of pairs (X,D), Internat. Math. Res. Notices (IMRN), Volume 2015, Issue 19, 9660-9695; arXiv:1302.1149. 21

[15] D. Iacono and M. Manetti: Semiregularity and obstructions of complete intersections, Advances in Mathematics 235 (2013), 92-125; arXiv:1112.0425. 9, 16

[16] S. Kobayashi: Differential geometry of complex vector bundles, Princeton Univ. Press (1987). 1

[17] M. Manetti: Lectures on deformations of complex manifolds, Rend. Mat. Appl. (7) 24 (2004), 1-183; arXiv:math.AG/0507286. 7, 15

[18] M. Manetti: Lie description of higher obstructions to deforming submanifolds, Ann. Sc. Norm. Super. Pisa Cl. Sci. 6 (2007), 631-659; arXiv:math.AG/0507287. 15, 16

[19] M. Manetti: Differential graded Lie algebras and formal deformation theory, In Algebraic Geometry: Seattle 2005. Proc. Sympos. Pure Math. 80 (2009), 785-810. 15, 16, 27

[20] M. Manetti: On some examples of obstructed irregular surfaces, Sci. China Math. 54 (2011), no. 8, 1713-1724. 2, 12

[21] H. Matsumura: Commutative Ring Theory, Cambridge Studies in Advanced Mathematics 8, Cambridge University Press, Cambridge, (1986). 5, 23

[22] S. Mukai: Symplectic structure of the moduli space of stable sheaves on an abelian or K3 surface, Invent. Math. 77 (1984), 101-116. 1, 2

[23] M. Schlessinger: Functors of Artin rings. Trans. Amer. Math. Soc. 130 (1968) 208-222. 16 
[24] T. Schürg. B. Toën, G. Vezzosi: Derived algebraic geometry, determinants of perfect complexes, and applications to obstruction theories for maps and complexes, J. Reine Angew. Math. 702 (2015); arXiv:1102.1150. 3

[25] E. Sernesi: Deformations of Algebraic Schemes, Grundlehren der mathematischen Wissenschaften, 334, Springer-Verlag, New York Berlin, (2006). 2, 3, 12, 22

[26] B. Teissier: The hunting invariants in the geometry of discriminants, in: (P. Holm editor) Real and complex singularities, Oslo (1976), 565-678. 11

[27] R. Thomas: A holomorphic Casson invariant for Calabi-Yau 3-folds, and bundles on K3 fibrations, Jour. Diff. Geom. 54 (2000), no. 2, 367-438. 1, 26

Università DEGLI StUdi Di BARI,

Dipartimento di Matematica,

Via E. Orabona 4, I-70125 Bari, Italy.

E-mail address: donatella.iacono@uniba.it

$U R L$ : www.dm.uniba.it/ iacono/

Università DEGLi STUdi di Roma "LA SAPIENZA",

Dipartimento di Matematica "Guido Castelnuovo",

P.le Aldo Moro 5, I-00185 Roma, Italy.

E-mail address: manetti@mat.uniroma1.it

$U R L$ : www.mat. uniroma1.it/people/manetti/ 